\title{
Study on 4PL as coordinating and constructing agent for supply chain systems: transaction cost theory approach and wave-particle duality
}

\author{
Guojun $\mathrm{Ji}^{*}$
}

\begin{abstract}
This paper introduces a new mathematical model for analyzing the economic benefits of incorporating the fourth party logistics (4PL), which is a contractor (i.e. agent) for the supply chain coordination and construction based on the division of community and the outsourcing development. Based on the physical theory and the wave-particle duality, a supply chain is the special organization whose characteristic has wave-particle duality. The mathematical model enriches the connotation of 4PL and it broadens the thought for 4PL development. Secondly, the proposed mathematical model predicated on transaction costs, is supported by Transaction Cost Theory (TCT) and acts as the theoretical analysis tool of 4PL for coordinating 3-party generic supply chain. Through the model, some trendy conclusions can be drawn to provide theoretical support for 4PL's practices. Finally, a case illustrates our conclusions.
\end{abstract}

Keywords: Fourth party logistics, Supply chain, Transaction cost theory, Coordination, Waveparticle duality. 


\section{Introduction}

The competition has become more and more drastic in globalization and represent among supply chains. Moreover, the competition transforms the coordinative pattern between supply chains from the conventional opposed pattern between enterprises. Hence, the integration and coordination of a supply chain (SC) are focused by its members. Generally, a core enterprise, called the 'Chain Lord', will be as the leader of the SC and act as organizing and coordinating the whole SC. This leader usually has the strong capability, such as supply capability, manufacture capability, or marketing capability. However, small and medium-sized enterprise (SME) is in the majority in market, e.g. in the China. The SC without such leader, based on these enterprises, is often lack of strong capability. Herein, using by an 'intermediator' to organize and coordinate is seemly choice. Therefore, the Fourth-Party Logistics (4PL) will act as intermediator in course of integrating SC. In fact, 4PL has itself predominance also plays vital role in the SC, such as offering information service or counseling etc. From European survey, 4PL's turnover increases at 10\% speed every year, Ryder and UPS are all developing their 4PL's ability. The definition of 4PL was introduced and registered as a brand by Accenture in 1996. A 4PL provider is a SC integrator that assembles and manages the resource, capacities, and technology of its own organization with those of complementary service providers to solve a comprehensive SC solution. The 4PL was developed by Accenture in 2002, that meant there are four factor for the successful 4PL (Bumstead, J., \& Cannons, K., 2002), i.e., Architect and Integrator, Intelligence Control Room, Supply-chain Infomediary, Resource Providers. Therefore, 4PL works with its customers, it can analyzes, model, integrate, control, and supervise some flows or SCs. Those will show 4PL lie in a higher position in the logistics operational network of the entire SC. In fact, 4PL supplies the integrated outsourcing services (Thomas Craig, 2003). The main characteristics are synthesized by Gattorna, J. \& Selen, W (2004) as follows: SC perspective layout, SC planner and optimization, transaction figurer and maker, SC reengineer, project management, service and system or information integrator, continuing innovator. However, most of 4PLs are developed with other professional logistics service suppliers' development. These are contained third party logistics, SC management consulting company and SC information technological company etc. Adam Smith suggested that productivity would rise significantly when the division of labor principle was used. Output per worker would be raised while costs per unit produced would be reduced. Division of social was caused more and more enterprises appeared, for example, enterprise function is more specialization, enterprise information is divided, then there are a mass of asymmetrical information among those enterprises. Such asymmetrical information results in more complication among them. Enterprises must waste the relative cost to communicate and coordinate with customers from upstream and downstream. 4PL acts as the integrated service supplier of SCs, it participates in the coordinative task and that not SC operation (Gattorna, J. \& Selen, W, 2004). By way of the SC' coordinator, 4PL may not hold facilities (such as warehouses, vehicles) and apply itself competence to deal with the coordinative work. For the concentrated SC organized by a core enterprise, 4PL can bear most of the coordinative tasks. Furthermore, 4PL has the professional coordination function, the coordinative effect may be better than the core enterprise does. For the decentralized SC organized 
by the nor powerful enterprise, $4 \mathrm{PL}$ will become a leader or agency of the $\mathrm{SC}$, to organize and coordinate whole SC operation, then united superintend under specialization. Therefore, 4PL supplies the comprehensive integration service to their customers, and lightens the coordinative burden. However, the prominent effect and function for 4PL is to integrate the SCs, which beyond two stages, multi-stage modes that may be included supplier, manufactory, retailer, reverse activities organizer and third party logistics provider practically. Herein, a newly definition of 4PL represents a contractor of the SC coordination in this paper. There are many researchers noticed the coordination mechanism of SC, such as 8 coordination mechanisms (Martinez, 1989); a SC coordination involves two levels in general coordination and multi-plant coordination (Bhatnagar, 1993); a SC coordination exists three types: buyer vendor coordination, production distribution coordination and inventory distribution coordination (Thomas, 1996); there are five coordination mechanisms: mutual adjustment, direct supervision, standardization of work output, standardization of work process and standardization of skills (Mintzberg, 1979) etc.

For the purpose of this study, 4PL is treated as a contractor of the SC integrator, inclusive of organizing and coordination SC operations. The prominent effect and function for 4PL is to integrate the SC, which constitute multi-stage modes: supplier, manufacturer, retailer and third party logistics provider. These member enterprises (or parties) of a typical SC have their own independent decision-making ability (Chopra, S., Meindl, P., 2001). Extant SC management literature posit that for maximizing the benefits of the whole SC, 'Supply Chain Coordination' is one of the core research issues. Accordingly, different levels of the SC coordination systems or the coordination systems among enterprises were introduced and developed. For example, Virtual Enterprises and Strategic Alliance have arisen in the macro-level coordinative organization among enterprises, as they provide a great deal of the theoretical reference in the SC coordination.

The SC costs generally consist in two main components: the production cost and transaction cost based on new institutional economics. Where the production costs are happened in the relationship between human and nature, production costs are typically easier to define and measure than transaction costs. Nevertheless, transaction costs are often acknowledged to be important and they are critical components of the total economic costs for a particular enterprise in a given industry. Williamson (1991) suggested that transactions costs have two components: ex ante and ex post economic costs. Ex ante economic costs include: (1) search and information costs, (2) drafting, bargaining and decision costs, and (3) costs of safeguarding an agreement. Ex post economic costs include: (1) costs of measuring input, (2) costs of measuring output, (3) monitoring and enforcement costs, and (4) adaptation and haggling costs. With the global economic development, the degree of professionalism for an enterprise will be consolidated by the division degree of sociality which will be positively enlarged. Intensifying specialization will lead to reducing production costs, so modern enterprises implement product differentiation strategies to gain competitive advantages. Consequently enlarging division degree will result in the affiliated enterprises (appeared in the entire life cycle of one product) implementing product differentiation strategy, i.e., the number of SC hierarchy goes up that causes the transaction time increases evidently, resulting in the increase of social transaction costs. From the transaction cost theory, there exists mismatch of coordination aim in SC due to 'each does things in their own way' among members. 
To achieve SC operation objectives based on lower cost, there must be a better organization and stimulating mechanism. From costs perspective, reducing production costs is determined within enterprise, controlled by member enterprises themselves. But, reducing transaction costs is the shared responsibility among enterprises in the SC, individual enterprise can not effectively achieve the goal, so it often has to be settled by an dedicated agent who can then effectively integrate and coordinate all members in the SC. In fact, in the information economy of today, the transaction costs of a 3-party SC dominated the whole costs, thus the main objective of SC coordination will focus on the transaction costs, which is well supported by literature. For example, Hobbs and Jill (1996) have discussed the theoretical framework of SC transaction costs, Grover and Malhotra (2003) raised an operation and management framework using transaction costs analysis, and concluded that the following problems could be analyzed based on the transaction cost theory: outsourcing and manufacturing/purchasing decision-making, investment distribution, coordination, integration, distribution in a SC etc. Therefore, the transaction cost theory can be adapted to analyze 4PL as agent for coordinating and constructing SC.

This paper organization is as follows: Based on the physical theory and the wave-particle duality, a SC is the special organization whose characteristic has wave-particle duality is discussed in section 2; In section 3, a mathematical model, based on transaction costs, is established by using Transaction Cost Theory as the theoretical analysis tool of 4PL coordinating SC. Through the model development process, some conclusions are drawn to provide theoretical support for 4PL's practices. In section 4, a case study illustrates our conclusions.

\section{Wave-particle duality and entanglement of quantum of supply chains}

\subsection{Wave-particle duality of a supply chain}

In allusion to dispute for light is 'wave' or 'particle' has lasted out more than 300 years, since 'wave theory' and 'particle theory' of light is opposite, the final result ascribes light is neither wave nor particle, and is the substance of wave and particle duality properties. If we call a 'SC' is 'wavemotion', and enterprises are 'particle-motion', then separation of a SC and enterprises is similar to antinomy for light is wave or particle on earth. The wave-motion of a SC means that different node enterprises integrated result. There are two describing variables for 'wave-motion', i.e., 'nodes' and 'nodes centralized degree'. With more the social division fine, more complicated and length the SC is. Essence of the SC's wave-motion is that process of 'explicit transmission' of the tangible semifinished product, return product and 'implicit transmission' of the intangible assets (e.g., information, brand, knowledge), which transmission scope is between semifinished product market and final product market or within SC, as well as it is the process of SC innovation and value realization. In such transmission process, there exists the definite fluctuation like as 'wave-motion' among every SC's nodes, so the SC can be described as 'wave-motion'. Otherwise, the organizational model of a SC is a network structure formed by upstream and downstream correlative enterprises. Every enterprise is just like as a 'particle' in a SC. There are two describing 
variables for 'particle-motion', i.e., 'node position' and 'innovation ability'. Enterprises often are changing in a SC's node position, then results in any SC is changing. And enterprises whether or not find, search, hold or change their node position to reach 'robustness' of the SC, which entirely depends on their innovation ability. Movement or determination of the node position together with magnitude of innovation ability, are closely relative to cooperation and competitive competence of a SC. Therefore, the 'particle-motion' denotes the integration competence of different enterprises along with a SC.

In quantum physics, 'quantum particle' and 'quantum wave' is uniform, quantum of particle must depends on 'wave-motion', and quantum of wave must depends on 'particle-motion' too (Shifan Wang, 2007). In fact, 'wave-motion' and 'particle-motion' in a SC is uniform. 'Particlemotion' indicates the production innovation of a SC or enterprises is drive of the integrated innovation and value actualization. The 'wave-particle duality' of a SC shows the chain is an organization by way of social division and the division coordination to come true, which is integration of enterprises, to implement the effective disposition resources and operation in the SC, and ensure division and collaboration favoringly. Based on the quantum physics, the Bohr atomic model has three hypotheses, i.e., energy level, jump and orbit. Correspondingly, the Bohr atomic model of a SC also has three hypotheses as follows: (1) Energy level. Enterprises in a SC always are in a series of discontinuous innovative states, synchronously the product in a SC or its enterprises is in a series of discontinuous value state too, such case is called stationary state. Transformation from one state to another often needs definite energy, so this will engenders the different energy levels. (2) Jump. Transformation of different enterprises or different product from one stationary state to another usually needs definite energy, in order to offset their shortage of technical and innovative competence, so this likes as jump. At the same time, the process of increase or decrease value likes as jump too. (3) Orbit. What is called orbit means nodes of a SC. There exists energy of technical innovation and difference value among the nodes of a SC, those nodes often are discontinuous.

Therefore, the meaning of the model is interpreted as follows: (i) Nucleus can denote the final product enterprises. In Bohr atomic model, proton and neutron likes as the final product enterprises in a SC, there usually exists a large number of proton and neutron in the chain. (ii) Electron can show the matching enterprises, core enterprise or the matching bottleneck. In a SC, electrons located in orbit are semifinished product matching enterprises, which they are situated in the SC's nodes. Same node may has many enterprises, which form the different multi-level SCs, and today's SC can regards as the dynamic network formed by several multi-level SCs. (iii) Orbit can denote node or matching radius. Orbit in a SC likes as nodes. Based on these orbits are away from nucleus distance difference, they in turn can hanger together from far till close to form the SC. Since nodes often are located in different position of the SC, if technical innovation competence is stronger and value-added is greater, the 'energy level' is higher. (iv) 'Quantum jump' can denote displacement of the semifinished product. The semifinished product between node orbits can move via forward and reverse logistics activities namely 'quantum jump'. 'Quantum jump' shows semifinished product along with its SC to pursues consummate continuously, transmission often is from low-node ( far from the final enterprise) to high-node (close with the final enterprise), then the $\mathrm{SC}$ can implement the whole 'jump' process from $\mathrm{R} \& \mathrm{D}$ to production then to consumption. In 
nature, the 'quantum jump' process is process of innovation and value-added in a SC.

\section{2 'Entanglement of quantum' and 'quantum jump' in a supply chain}

In the quantum physics, 'entanglement of quantum' shows an phenomena, i.e., whether how far two particles, one particle's change will affect another, they are interactional radically (Dekui Wang, 2004). There is an especial 'entanglement of quantum' phenomena in a SC, whether government or enterprises themselves, are all encircled some of enterprises like as 'entanglement of quantum', then they foster and optimize the SC. 'Entanglement of quantum' indicates the relationship between SC and enterprises, which they are neither the entire independent market transaction relations, nor the relative close interior relations within the SC, and is a 'keep it at an arm's length' entanglement relation, such relation shows change of one enterprise must affect other enterprises even the entire chain, so it may presents 'entanglement of quantum'. Change in 'entanglement of quantum' incarnates the change of 'quantum state'. In the quantum physics, 'quantum state' denotes the state of particles (such as atom, neutron, proton), can represents energy, rotation, motion, magnetic field of particles and other physical characteristics (Dekui Wang, 2004). 'Quantum state' in a SC can present the state of 'implicit knowledge' in enterprises. Implicit knowledge is enterprises own knowledge, technology, brand, information etc. In the quantum physics, study on 'entanglement of quantum' objective is to reach 'implicit transmission' of quantum information. So, study on the SC's objective is to actualize 'implicit transmission' of quantum states of enterprises. What is called 'implicit transmission' that shows just to transmit carriers (semifinished product or product) of technology, information, brand, value etc., does not transmit themselves. In the SC, 'entanglement of quantum' objective of enterprises is to implement 'implicit transmission' of technology, band and so on. The explicit shape of the SC is the 'explicit transmission' of semifinished product, and the implicit shape is the 'implicit transmission' of implicit knowledge. In addition, with increase of the production factor price and the global extension of SC, the government must understand its role (service, coordination or support), if it blindly issues some preference policies, the SC will be difficult to develop or optimize. Apparently, using by government's preference policies to attracting enterprises, the objective should form 'entanglement of quantum' and be easy to develop enterprises ultimately. In this way, a SC must depend on 'entanglement of quantum' requirements to build a perfect chain, or else, the chain relations will can't maintain stability and long-term.

In the quantum physics, to carry through long-distance quantum communication or quantum state implicit transmission, we must allow long-distance particles together with short-distance particles can hold maximal 'quantum entanglement state' (Dekui Wang, 2004). Similarly, in a SC, we must try hard to impel the 'quantum entanglement state' formed among enterprises, i.e., such that 'quantum state' presents entanglement state. In addition, the technical standard to the SC's affection is self-evident, once a technical standard change, enterprises in the SC will change to fit a new standard. The process of constituting standard is the process of 'quantum entanglement state' formed. And the process of driving standard is the process of SC formed via the standard or implicit knowledge transmission. For example, Microsoft Inc. always perseveres in innovation over time. In fact, the technical innovative process is the process of 'quantum entanglement state', 
once the 'quantum entanglement state' engendered, it will drive enterprises change virtually, the 'entanglement of quantum' will appear, then results in further integration of the 'explicit transmission' of Microsoft Inc. and the chain. Nowadays, 4PL can collect plenty of information of the supply network in course of the supply chain evaluation and find the bottle-neck of the supply chain management. Therefore, 4PL can provide more consulting service and realize the integrative optimization of the supply chain.

In the quantum physics, because there are diversified unavoidable environmental noises in the communication channel, quality of the 'quantum entanglement state' will decrease with the transmission distance increase (Dekui Wang, 2004). In a SC, the quality of 'entanglement of quantum' among enterprises also decreases with their distance increase and environmental noises. In fact, many reasons can make the environmental noise of the SC, such as the social economic environment, business strategies, matching model of enterprises etc. Therefore, keep the 'quantum entanglement state' and achieve 'quantum jump' are correlative important to the SC. The process of 'quantum jump' is the process of the 'quantum entanglement state' kept.

\section{The transaction cost analysis models for 4PL coordinating supply chain}

From the macro viewpoint, SC coordination can be divided to decentralized and centralized. Decentralized refers to the SC coordination is voluntary organized by the enterprises in the SC, such mode is appeared in the industry group constructed by Small \& Medium Enterprise. Centralized mode means that the SC coordination is organized by a core enterprise (or Chain Lord), the core enterprise is the leader in its SC. Centralized mode is widely applied at present, e.g., Dell computers. In fact, the coordinative mode based on 4PL belongs to the centralized mode. We will analyze the transaction costs whether or not there is $4 \mathrm{PL}$ to coordinate a SC as follows.

Based on Williamson viewpoints, we think that the transaction costs of SC coordination are divided to ex ante costs and ex post costs. In order to set up our model, we give the following basic assumptions: (1) enterprises at the same hierarchy in the SC have same production costs; (2) the constructed SC is a cluster, i.e. all member enterprises in the SC implement single shared strategy; (3) there is only one 4PL that participates SC operation. Variables considered in the proposed mathematical model are as follows: $m$ denotes that general hierarchy in a SC system; $n_{i}$ denotes the selected enterprises of $i$ hierarchy in a SC system; $s$ represents the selected 4PL in a SC system.

\subsection{The transaction cost models based on the decentralized supply chain coordination}

In the decentralized SC, all member enterprises search information using top-down approach, i.e. from super-hierarchy or down-hierarchy enterprises respectively. The outcome of search information process leads to the selection of performance standard and then sign up a series of contracts, for subsequent construction of a SC, see Figure 1 (a). After constructed the SC, enterprises coordinate with super-hierarchy or down-hierarchy enterprises respectively and implement the SC operation, see Figure 2 (a). Figures 1 (b) and 2 (b) represent the SC system structure before and 
after the establishment of decentralized supply-chain coordination respectively. The selected 4PL will take on the coordinative tasks in the constructed SC system. To the decentralized SC, the transaction costs incurred in signed contract (ex ante) can be expressed as:

$$
C_{a}=\sum_{i=1}^{m} \sum_{j=1}^{n_{i}}\left[\sum_{k=1}^{n_{(i-1)}} a_{i j k}+\sum_{l=1}^{n_{(i+1)}} a_{i j l}\right]
$$

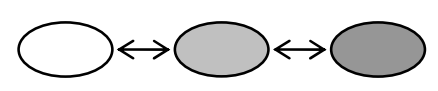

a. Decentralized SC system structure (before the SC constructing)

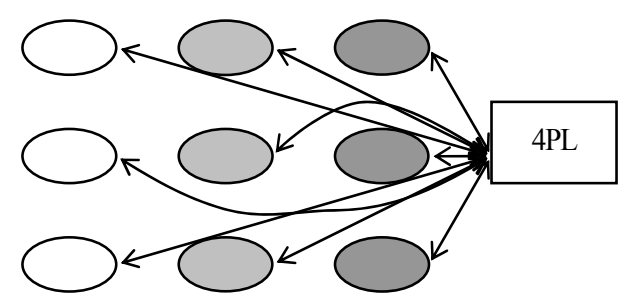

(i-1) hierarchy

$i$ hierarchy

(i+1) hierarchy

b. Decentralized SC system structure based on 4PL (before the SC constructing)

Figure 1. Decentralized SC system structure fore-and-aft 4PL entered (before the SC constructing)

where, $a_{i j k}$ denotes $i$ hierarchy $j$ enterprise's committed ex ante transaction costs between $i$ hierarchy $j$ enterprise and $(i-1)$ hierarchy $k$ enterprise in the SC; $a_{i j l}$ denotes $i$ hierarchy $j$ enterprise's committed ex ante transaction costs between $i$ hierarchy $j$ enterprise and $(i+1)$ hierarchy $l$ enterprise in the SC. Thus $\sum_{k=1}^{n(i-1)} a_{i j k}+\sum_{l=1}^{n(i-1)} a_{i j l}$ represents $i$ hierarchy $j$ enterprise's committed ex ante transaction costs.

To the decentralized SC, the transaction costs incurred in the signed contract (ex post) can be expressed as

$$
C_{p}=\sum_{i=1}^{m} p_{i}
$$

where, $p_{i}$ denotes the ex post transaction economic costs happened in $i$ hierarchy enterprise with upper and lower hierarchy enterprises. Then, the transaction costs of decentralized SC are given by:

$$
C=C_{a}+C_{p}
$$




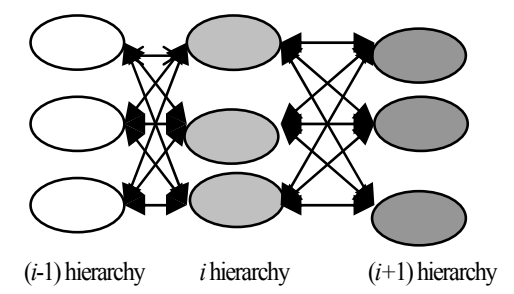

a. Decentralized SC system structure (after the SC constructed)

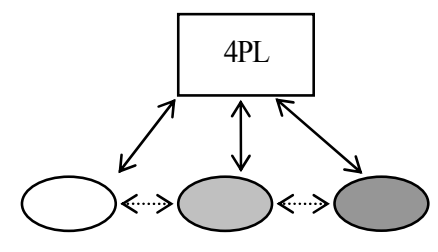

b. Decentralized SC system structure based on 4PL (after the SC constructed)

Figure 2. Decentralized SC system structure for-and-aft 4PL entered (after the SC constructed)

Once there is 4PL entering the decentralized SC, such SC will become a centralized SC, the ex ante transaction costs incurred before the SC construction are given by:

$$
C_{a}^{F}=\sum_{i=1}^{S} a_{i}+\sum_{i=1}^{m} \sum_{j=1}^{n_{i}} a_{i j F}+\sum_{i=1}^{m} \sum_{j=1}^{n_{i}} a_{F i j}
$$

where, $a_{i}$ denotes $i$ PL ex ante transaction costs when 4PL is selected; $a_{i j F}$ denotes $i$ hierarchy $j$ enterprise's committed ex ante transaction costs between 4PL and $i$ hierarchy $j$ enterprise; $a_{F i j}$ denotes 4PL's committed ex post transaction costs between $i$ hierarchy $j$ enterprise and 4PL.

At the same time, when 4PL entering the decentralized SC, the ex post transaction costs incurred after the SC is constructed can be expressed:

$$
C_{p}^{F}=\sum_{i=1}^{m} q_{i}+\sum_{i=1}^{m} p_{i F}+\sum_{i=1}^{m} p_{F i}
$$

where, $q_{i}$ denotes the ex post transaction costs happened in $i$ hierarchy enterprise with upper and down hierarchy enterprises (under 4PL took on the coordinative tasks), such costs happened in 4PL can not bear ex post coordinative works; $p_{i F}$ denotes the ex post transaction costs happened in $i$ hierarchy enterprise committed costs between 4PL and $i$ enterprise; $p_{i F}$ denotes the ex post transaction costs happened in 4PL committed costs between 4PL and $i$ enterprise.

Therefore, the ex ante and ex post economic transaction costs can be described respectively as, regardless whether 4PL has entered SC or not.

$$
\Delta C_{a}=C_{a}-C_{a}^{F}=\sum_{i=1}^{m} \sum_{j=1}^{n_{i}}\left[\sum_{k=1}^{n_{(i-1)}} a_{i j k}+\sum_{l=1}^{n_{(i+1)}} a_{i j l}-a_{i j F}-a_{F i j}\right]-\sum_{i=1}^{S} a_{i}
$$




$$
\Delta C_{p}=C_{p}-C_{p}^{F}=\sum_{i=1}^{m}\left(p_{i}-q_{i}-p_{i F}-p_{F i}\right)
$$

and, the transaction economic costs when 4PL has entered are represented as follows:

$$
\Delta C=\Delta C_{a}+\Delta C_{p}
$$

Without loss of generality, suppose that $n_{i}=n, a_{i j k}=a_{i j l}=a, a_{i j F}=a_{F i j}=a_{F}, a_{i}=b, p_{i}=p$, $q_{i}=q, P_{i F}=P_{F i}=P_{F}$, then, (6), (7) and (8) can be formulated respectively as:

$$
\begin{aligned}
& \Delta C_{a}=2 m n\left(n a-a_{F}\right)-s b \\
& \Delta C_{p}=m\left(p-q-2 p_{F}\right) \\
& \Delta C=\Delta C_{a}+\Delta C_{p}=m\left(2 a n^{2}+p-2 n a_{F}-q-2 p_{F}\right)-2 s b
\end{aligned}
$$

Considering 4PL characteristics, it has information search ability and the professional negotiation ability for constructing SC, hence, the sense level for 4PL constructed SC is usually higher than other members; 4PL constructs effective SC based on integrated view, often hold complete information and alleviated asymmetrical information influence, and thus reduces the opportunism appearance; 4PL is effective at the SC coordinating work that results in the special assets being used repeatedly, thereby, lower the special assets investment risk; holding the comprehensive information and profuse constructing SC experience, then 4PL has powerful ability to respond to uncertainty. Consequently, 4PL uses lesser transaction economic costs for the operation of the constructed SC. For example, if $a \geq a_{F}$, then $n a \geq a_{F}$ such that $2 m n\left(n a-a_{F}\right) \geq 0$. And that, $a<a_{F}$, as long as $n a \geq a_{F}$, still guarantee that $2 m n\left(n a-a_{F}\right) \geq 0$ holds, this is due to SC structure changed. As well as, $s b \geq 0$, means that $n a>a_{F}$, and only if $2 m n\left(n a-a_{F}\right)>s b$, we have $\Delta C_{a}>0$, i.e., if and only if the committed transaction economic costs are lower when 4PL entering constructed SC, 4PL just can take on the SC construction tasks.

In addition, 4PL has vast experience in ways of $\mathrm{SC}$ integrating operation and excellent manpower utilization, so 4PL has higher sense level than other members; 4PL acts as information platform in the SC operation, so it can alleviate the asymmetrical information effectively, improve the SC operation efficiency, herein it ensures furthest information sharing and is the preferable means to deter opportunism appearance. In short, $4 \mathrm{PL}$ commits much less transaction economic costs to construct a SC. For example, if $p \geq q+2 p_{F}$, then we have $\Delta C_{p}>0$, i.e., when $4 \mathrm{PL}$ enters the SC, it can lower the transaction economic costs effectively in the SC operation, besides undertake the $\mathrm{SC}$ coordinating works.

Suppose $\Delta C_{a}<0$ or $\Delta C_{p}<0$, but if only $\Delta C>0$, i.e., lowering the entire transaction costs in a $\mathrm{SC}$ after 4PL entered, then 4PL can still be competent for the SC coordinating tasks. From (9), (10) and (11), when $\left(n a-a_{F}\right)>0$ (4PL can economize the ex ante transaction costs in all levels of the 
$\mathrm{SCs}$ ), it is easy to see that $\Delta C_{a}$ is in direct ratio to $m$, then $\Delta C_{a}$ will increase along with the SC system hierarchy add on, and there is a critical point $K_{1}$ such that if $m \geq K_{1}$, then $\Delta C_{a} \geq 0$, and $K_{1}$ satisfies:

$$
K_{1}=\frac{s b}{2 n\left(n a-a_{F}\right)}
$$

If $\left(p-q-2 p_{F}\right)>0$ (4PL can economize the ex post transaction costs in all levels SCs), $\Delta C_{p}$ is in direct ratio to $m$, then $\Delta C_{p}$ will increase along with the SC system hierarchy add in; when $\left(2 a n^{2}+p-2 n a_{F}-q-2 p_{F}\right)>0$ (4PL can economize the transaction costs in all levels of the SCs), $\Delta C$ is in direct ratio to $m$, then $\Delta C$ will increase with the SC system hierarchy add in, and there is a critical point $K_{2}$, such that if $m \geq K_{2}$, then $\Delta C \geq 0$, and $K_{2}$ satisfies:

$$
K_{2}=\frac{s b}{\left(2 a n^{2}+p-2 n a_{F}-q-2 p_{F}\right)}
$$

We can find that increasing $\Delta C_{a}$ and $\Delta C_{p}$ support $4 \mathrm{PL}$ enlarging the profit space fore-and-aft in the constructed SC. In fact, with the social division becomes more significant, the whole SC hierarchy will be expanded, thus 4PL entering SC systems probability will increase.

The expression (9) can be translated into as follows:

$$
\Delta C_{a}=2 m a\left(n-\frac{a_{F}}{2 a}\right)^{2}-\frac{m a_{F}^{2}}{2 a}-s b
$$

It is easy to see that $\Delta C_{a}$ is a quadratic function about $n$ implying that the same hierarchy enterprises in the SC also increases, resulting to $\Delta C_{a}$ increases rapidly, this is due to two different Sc systems. When 4PL entering SC, it can change the system structure, enterprises' contact time decreases obviously, so will reduce negotiation times, lower the search costs among enterprises, and economizing the ex ante transaction costs largely. There exists a critical value $K_{3}$, if $n \geq K_{3}$, then $\Delta C_{a} \geq 0$, here $K_{3}$ satisfies:

$$
K_{3}=\frac{a_{F}}{2 a}+\sqrt{\frac{m a_{F}^{2}+2 a s b}{2 m a^{2}}}
$$

\subsection{The transaction cost models based on the centralized supply chain coordination}

In the centralized SC, there is a core enterprise that takes charge all search members of the SC based on the determined standard, and constructs a SC by using the series of the signed contracts, 
see Figure 3 (a). After constructed the SC, 4PL assumes the coordination role for the entire SC operation, see Figure 4 (a).

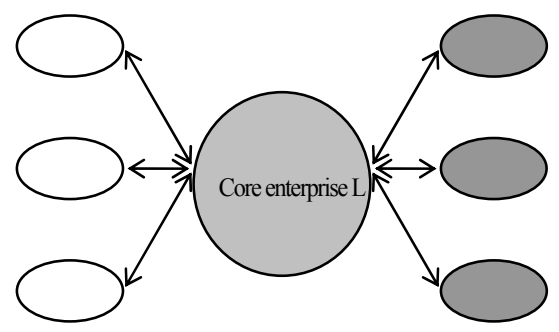

a. Centralized SC system structure (before SC constructing)

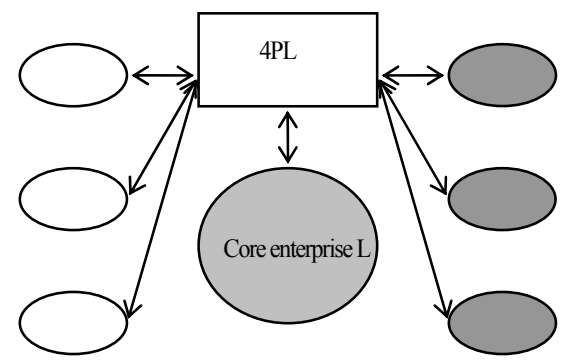

b. Centralized SC system structure contained 4PL (before SC constructing)

Figure 3. Centralized SC system structure fore-and-aft 4PL entered (before the SC constructing)

To the centralized SC, the transaction costs incurred in the signed contract (ex ante) satisfy as follows:

$$
C_{a}=\sum_{\substack{i=1 \\ i \neq L}}^{m} \sum_{j=1}^{n_{i}}\left(a_{i j L}+a_{L i j}\right)
$$

where $a_{i j L}$ denotes $i$ hierarchy $j$ enterprise's committed ex ante transaction costs between $i$ hierarchy $j$ enterprise and the core enterprise in a SC; $a_{L i j}$ denotes the core enterprise's committed ex ante transaction costs between $i$ hierarchy $j$ enterprise and the core enterprise. 


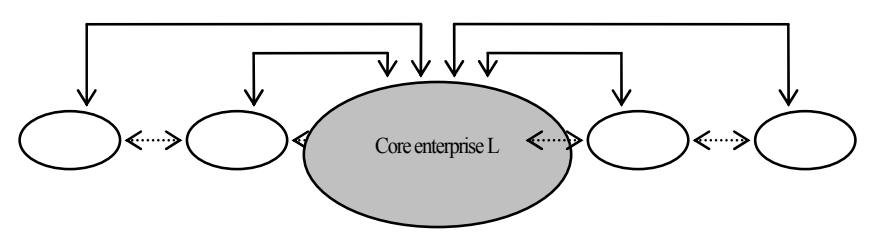

a. Centralized SC system structure (after SC constructed)

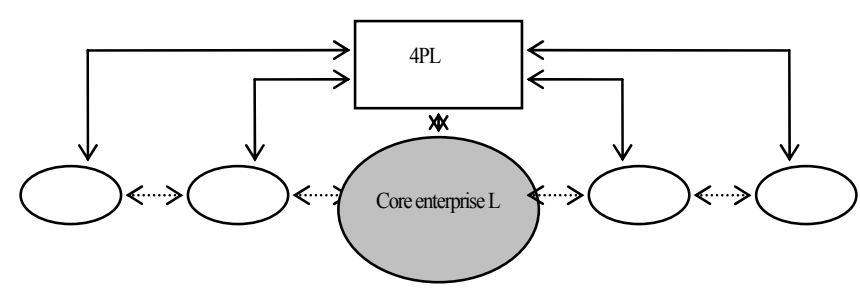

b. Centralized SC system structure contained 4PL (after supply chain constructed)

Figure 4. Centralized SC system structure fore-and-aft 4PL entered (after the SC constructed)

To the centralized SC, the transaction costs committed in signed contract (ex post) satisfy as follows:

$$
C_{p}=\sum_{\substack{i=1 \\ i \neq L}}^{m}\left(p_{i L}+p_{L i}\right)+\sum_{i=1}^{m} p_{i}
$$

where, $p_{i L}$ denotes $i$ hierarchy committing the ex post transaction costs when the core enterprise coordinating $i$ hierarchy enterprises; $p_{L i}$ denotes the core enterprise's committed ex post transaction costs when the core enterprise coordinating $i$ hierarchy enterprises; $p_{i}$ denotes $i$ hierarchy enterprise's committed ex post transaction costs produced in upper and down enterprises.

In this way, the transaction cost in the centralized SC is as follows:

$$
C=C_{a}+C_{p}
$$

When 4PL entered SC system, the selected 4PL will substitute the core enterprise to constructing SC and bear the coordinating work in the constructed SC.

When 4PL entered, the transaction costs before SC constructing (ex ante) satisfy:

$$
C_{a}^{F}=\sum_{i=1}^{m} \sum_{j=1}^{n_{i}}\left(a_{i j F}+a_{F i j}\right)+\sum_{i=1}^{s}\left(a_{i L}+a_{L i}\right)
$$


where, $a_{i j F}$ denotes $i$ hierarchy $j$ enterprise's committed ex ante transaction costs incurred between 4PL and $i$ hierarchy $j$ enterprise in a SC system; $a_{F i j}$ denotes 4PL committed ex ante transaction costs incurred between 4PL and $i$ hierarchy $j$ enterprise in a SC system; $a_{i L}$ denotes the selected $i$ 4PL's committed ex ante transaction costs incurred between $i$ 4PL and the core enterprise; $a_{L i}$ denotes the core enterprise's committed ex ante transaction costs produced between $i$ 4PL and the core enterprise.

When 4PL entered SC system, the transaction costs committed in the constructed SC (ex post) are represented:

$$
C_{p}^{F}=\sum_{i=1}^{m}\left(p_{i F}+p_{F i}\right)+\sum_{i=1}^{m} q_{i}
$$

where, $p_{i F}$ is $i$ hierarchy enterprises' committed ex post transaction costs when 4PL is coordinating $i$ hierarchy enterprises; $p_{F i}$ is $4 \mathrm{PL}$ 's committed ex post transaction costs when $4 \mathrm{PL}$ is coordinating $i$ hierarchy enterprises; $q_{i}$ is $i$ hierarchy enterprises committing the ex post transaction costs produced between 4PL and upper and down hierarchy enterprises.

Moreover, when 4PL entered SC or not, the ex ante transaction economic costs and the ex post transaction economic costs are as follows, respectively:

$$
\begin{aligned}
& \Delta C_{a}=C_{a}-C_{a}^{F}=\sum_{\substack{i=1 \\
i \neq L}}^{m} \sum_{j=1}^{n_{i}}\left[a_{i j L}+a_{L i j}-a_{i j F}-a_{F L i j}\right]-\sum_{i=1}^{S}\left(a_{i L}+a_{L i}\right) \\
& \Delta C_{p}=C_{p}-C_{p}^{F}=\sum_{\substack{i=1 \\
i \neq L}}^{m}\left(p_{i L}+p_{L i}-p_{i F}-P_{F i}\right)-\sum_{i=1}^{m}\left(p_{i}-a_{i}\right)-\left(p_{F L}+p_{L F}\right)
\end{aligned}
$$

Therefore, the transaction economic costs when 4PL entered SC satisfy:

$$
\Delta C=\Delta C_{a}+\Delta C_{p}
$$

Without loss of generality, suppose $n_{i}=n, a_{i j L}=a_{L i j}=a_{L}, a_{i j F}=a_{F i j}, a_{i L}=a_{L i}=c, p_{i L}=p_{L i}=p_{L}$, $p_{i F}=p_{F i}=p_{F}, p_{i}=p, q_{i}=q, p_{F L}=p_{L F}=t$, then expressions (21), (22) and (23) can be simplified as follows, respectively:

$$
\begin{aligned}
& \Delta C_{a}=2(m-1) n\left(a_{L}-a_{F}\right)-2 s c \\
& \Delta C_{p}=2(m-1)\left(p_{L}-p_{F}\right)+m(p-q)-2 t
\end{aligned}
$$




$$
\begin{aligned}
\Delta C=\Delta C_{a}+\Delta C_{p} & =m\left(2 n a_{L}+2 p_{L}+p-2 a_{F}-2 p_{F}-q\right) \\
& -2\left(n a_{L}+p_{L}-a_{F}-p_{F}\right)-2 s c-2 t
\end{aligned}
$$

Similar to the discussion in section 2.1, it is easy to see that $a_{L} \geq a_{F}$ satisfies averagely. Since $s c \geq 0$, if $a_{L}>a_{F}$ and such that $2(m-1) n\left(a_{L}-a_{F}\right)>2 s c$ eventually, we just have $\Delta C_{a}>0$, i.e., when $4 \mathrm{PL}$ entered SC and can lower the transaction costs incurred in the constructed hierarchy, thus justifying 4PL's undertaking the task of constructing SC.

In addition, it is easy to see that $p_{L} \geq p_{F}$ averagely. $p$ and $q$ are the coordinating costs committed in the core enterprise and 4PL can not substitute other members in the SC, if the difference between $p$ and $q$ is not too large, we can approximate $p=q$. Since $2 t \geq 0$, if $p_{L}>p_{F}$ and such that $2(m-1)\left(p_{L}-p_{F}\right)+m(p-q)>2 t$, we just have $\Delta C_{p}>0$, i.e., when 4PL entered $\mathrm{SC}$ and can lower the transaction costs incurred in the operation hierarchy, it is justifiable for 4PL to undertake the task of SC operation.

Suppose $\Delta C_{a}<0$ and $\Delta C_{p}<0$, but if only $\Delta C>0$, i.e., when 4PL entered SC and can lower the transaction costs incurred in the entire $\mathrm{SC}$, it is still justifiable for 4PL to undertake the task of SC coordination.

Similar to the decentralized SC, from (24), (25) and (26), when $\left(a_{L}-a_{F}\right)>0$ (4PL can economize the ex ante transaction costs in all levels of SCs), we can find $\Delta C_{a}$ is in direct ratio to $m$, i.e., along with the SC system hierarchy add in, $\Delta C_{a}$ can increase gradually, and there is a critical point $K_{4}$ such that when $m \geq K_{4}, \Delta C_{a} \geq 0$, and $K_{4}$ satisfies:

$$
K_{4}=\frac{s c}{n\left(a_{L}-a_{F}\right)}+1
$$

When $\left[2\left(p_{L}-p_{F}\right)+(p-q)\right]>0$ (4PL can economize the ex ante transaction costs in all levels of SCs), $\Delta C_{p}$ is in direct ratio to $m$, i.e., along with the SC system hierarchy add in, $\Delta C_{p}$ can increase gradually, and there is a critical point $K_{5}$ such that when $m \geq K_{5}, \Delta C_{p} \geq 0$, and $K_{5}$ satisfies:

$$
K_{5}=\frac{2\left(p_{L}-p_{F}\right)+2 t}{2\left(p_{L}-p_{F}\right)+(p-q)}
$$

When $\left(2 n a_{L}+2 p_{L}+p-2 a_{F}-2 p_{F}-q\right)>0$ (4PL can economize the ex ante transaction costs in all levels of SCs), $\Delta C$ is in direct ratio to $m$, i.e., along with the SC system hierarchy add in, we can find $\Delta C$ increase gradually, and there is a critical point $K_{6}$ such that when $m \geq K_{6}, \Delta C \geq 0$, and $K_{6}$ satisfies: 


$$
K_{6}=\frac{2\left(n a_{L}+p_{L}-a_{F}-p_{F}\right)+2 s c+2 t}{2 n a_{L}+2 p_{L}+p-2 a_{F}-2 p_{F}-q}
$$

We can find that increases of $\Delta C_{a}$ and $\Delta C_{p}$ support 4PL's enlarging of the profit space foreand-aft of the constructed SC.

From (24), we can know $m \geq 1$ generally, hence, if $\left(a_{L}-a_{F}\right)>0$ (4PL can economize the ex ante transaction costs in all hierarchy SCs), $\Delta C_{a}$ is in direct ratio to $n$, i.e., along with the $\mathrm{SC}$ hierarchy add in, we can find $\Delta C_{a}$ increases gradually, and there exists a critical point $K_{7}$ such that if $n \geq K_{7}$, then $\Delta C_{a} \geq 0$, and $K_{7}$ satisfies:

$$
K_{7}=\frac{s c}{(m-1)\left(a_{L}-a_{F}\right)}
$$

We can know that increases $\Delta C_{a}$ will support 4PL's enlarging the profit space before constructing the SC. However, since the SC system does not change too, not as good as the decentralized $\mathrm{SC}, \Delta C_{a}$ will increase along with $n$ rapidly.

By the transaction costs analysis for $4 \mathrm{PL}$ in coordinating $\mathrm{SC}$, we can find, no matter what $4 \mathrm{PL}$ coordinating the decentralized or centralized supply chains, the types of 4PL coordinating SC can be divided based on the operation types of supply chain contract signed. 4PL may do nothing but constructing SC, such as counseling service, agency platform service, cooperative members selecting service in supply chain etc.; 4PL can undertake the operation task in the construction of SC too, such as management service in the SC. Certainly, 4PL can undertake all fore-and-aft tasks simultaneously, but it needs to consider 4PL current ability. In addition, the transaction costs incurred in 4PL entered the decentralized SC are usually higher than those incurred in 4PL entered the centralized SC, hence, $4 \mathrm{PL}$ in the decentralized SC is economically more justifiable. Therefore, to earlier 4PL, it is easy to enter the decentralized SC, thus focusing on the development of the coordinating work to the decentralized SC. From the scale of SC (here, the scale determined $m$ hierarchy in a SC and the selecting enterprises $n_{i}$ ), scale of SC has deterministic effect on 4PL economizing the transaction costs of entire SC, i.e., larger scale of SC is favorable of 4PL's entering. With the social division becomes more and more detailed, the scale of supply chain will become enlarge, so 4PL has the cost advantage to entering SC in the future. Based on abovementioned, 4PL can combine its specialization and actuality, adopt the flexible strategy to develop, begin from SC coordination parts, and extend to coordinating the entire SC gradually. In fact, many 4PLs' development is based on such strategy.

\section{The supply chain coordination based on fractal theory}

Under the global and informatization market, a SC is on the dynamic surroundings, especially, 
short-term and medium-term SCs dynamic characteristics are obvious. However, long-term SCs are stabled in organization structures and collaboration relationships among their members, still there is determinate dynamic, e.g., the region market fluctuation. Therefore, coordination is very important to a SC. In this section, based on the fractal theory, we will analyze 4PL constructing SC coordination systems.

The term 'fractal' has been coined to describe complex organisms and structures in nature by using a self-similar characteristic (Warnecke, 1993). The architectural model of fractals represents a hierarchical structure built from elements of a basic fractal unit (BFU), and the design of a basic unit incorporates a set of pertinent attributes that can fully represent any level in the hierarchy (Tirpak et al.1992). In other words, the term 'fractal' can represent an entire organization at the highest level or an elementary unit at the bottom level. This paper basically adapts the formal definition of the fractal which was firstly proposed by Warnecke (1993) to solve general problems in a SC management. The fractal is defined as 'an independently acting corporate entity whose goal and performance can be precisely described.' To function as a coherent whole, however, goal consistency should be maintained through a goal-formation process that is supported by an inheritance mechanism (Tharumarajah et al. 1996). The fractal has intrinsic characteristics that include self-organization, self-optimization, goal-orientation, self-similarity, vitality and dynamics (Warnecke, 1993; Ryu et al., 2000). The BFU mainly consists of five functional modules: an observer, an analyzer, a resolver, an organizer and a reporter. It also has several auxiliary modules for helping operations of main modules, which can be adjusted with respect to application domains. Figure 5 illustrates the architecture of the bottom-leveled fractal and relationships among functional modules (Ryu et al., 2000).

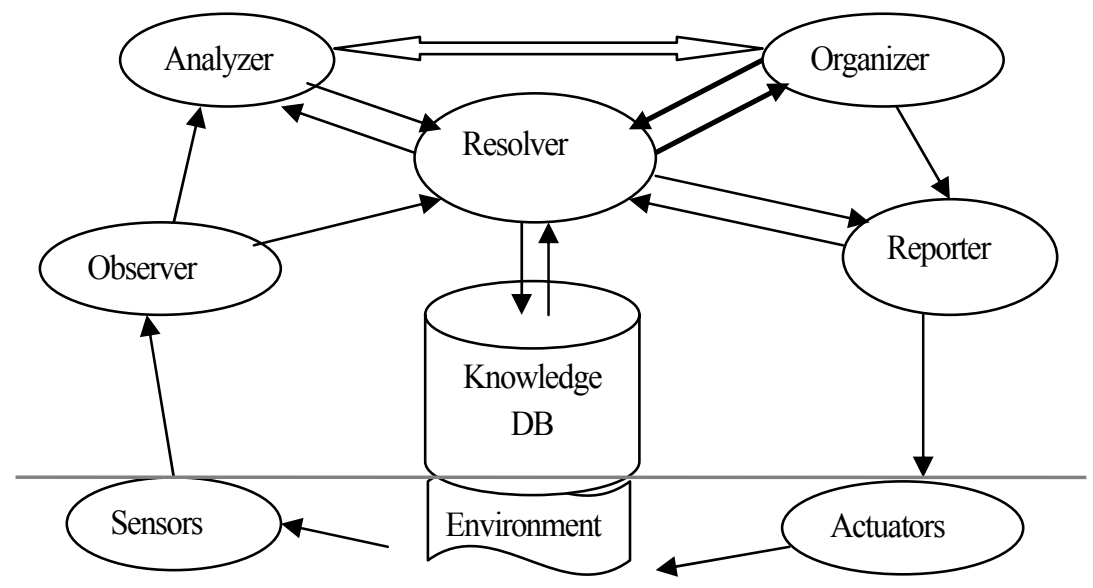

Figure 5. Fractal architecture and relationships among functional modules

According to abovementioned 4PLs characteristics, 4PL can be based on self-similarity of 
fractal-based SC, to construct coordinating BFUs of a SC. Such BFU has 'holography' relationship with the entire SC, i.e., any or any parts of BFU can be similar to whole (Tong Wu, 2001). Consistent with the fractal concept, each member of SC becomes a fractal, and any combination of each member can be another fractal. Ryu et al. (2002) proposed a fractal-based framework for the management of $\mathrm{B} 2 \mathrm{C}$ companies. Based on the 'holography' relation, using by 'fractal structure technique', the complex organisms can be represented a hierarchical structure and have selfsimilarity built from elements of a basic fractal unit (Fengbin Wang, 2005). The 'holography' relation results in BFUs of a SC have the coherent objectives and characteristics. 4PL can decompose the entire objectives together with all members in a SC, activities are actualized by BFUs. In any BFUs, because they have self-similarity of the fractal-based SC, there are definite homologous relationships among aspects, such as operation mode, enterprise culture, system platform, organization structure etc., so effective coordination can be easy to reach within BFUs. BFU has nested structure, see Figure 6. The entire SC makes of BFUs, any of BFUs have selfsimilarity at a certain extent, so can achieve the different coordination. 4PL can apply BFUs to take full advantage of characteristics (such as self-organization, selfoptimization), act as hierarchical structures coordinating units, and BFUs can integrate or separate dynamically, then the dynamic coordination architectural model can be constructed.

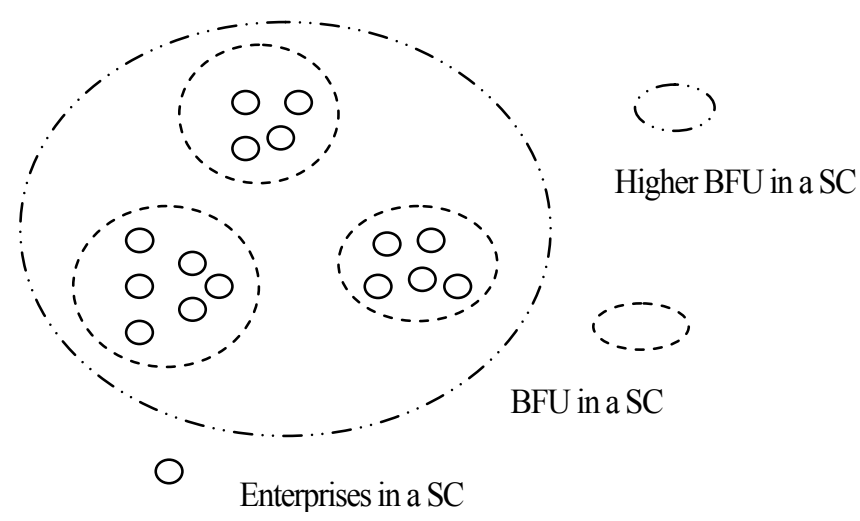

Figure 6. The nested structure of BFUs in a SC

4PL coordinating the fractal-based SC system is often driven via its objectives, according to the coordination requirements in the dynamic SC, realize standing optimal coordination control system under collaboration among 4PL and members in SC. The constructing steps of the system are expatiated as follows:

\subsection{Establishing and decomposing objectives, evaluating resources and restrictions}

Construction and operation of a SC always depend on its objectives, 4PL acts as a coordinator must understand its undertaking tasks in the SC. 4PL together with members in a SC, especially 
with the core members establishes the integrated objectives, and decomposes those objectives one by one, forms the different hierarchical sub-objective, then introduce the orientation for constructing the fractal-based SC. Establishing and decomposing objectives is confined by resources and other restrictions, so the fractal-based SC constructed by 4PL and members should carry with the relative activity in the definite fractal space. Objective, resource and restrictions together construct a threedimension fractal space, and fractal rules and coordination organization structure of the fractalbased SC are based on the space. When 4PL together with members of the fractal-based SC establishing and decomposing objectives, must insure all BFUs share the integrated objective, exert 'information platform' function adequately.

When 4PL constructing and decomposing objective of the fractal-based SC, evaluating resources and restrictions, it should adopt different strategies according to different types of SCs. To short-term SC, there exists higher uncertainty, BFU constructing frequency is higher, when 4PL coordinates BFUs, it together with members of the fractal-based SC must refine the objective and decompose objective one by one. Based on the refined objective, members in the fractal-based SC sign the detailed relative contact, declare their right and responsibility, these are convenient for renewing work as soon as possible while all BFUs regulated widely or BFUs disorganized. To medium-term SC, comparing with short-term, endogenous uncertainty will reduce, results in reengineering frequency lowering for BFUs in the fractal-based SC, 4PL should gradually shift its pivot work to help establish close faith relationship among members in the fractal-based SC, and set up the long-term league's vision, to reply all kind of endogenous uncertainty in future cooperation.

\subsection{Constructing BFUs}

BFUs construction is based on self-similarity of the fractal-based SC, just about such characteristic will result in the SC is in stable state relatively. Constructing BFUs will be based on higher similarity unit to manage to harmonious consistency easily, and the coordinating costs may be lower. Furthermore, BFU is also a dynamic organization, can update its structure continuously.

\section{Constructing process}

Firstly, 4PL constructing BFU is based on the integrated objective and sub-objective to determine fractal rules, i.e., all BFUs comply with the main similarity when constructing BFU. The generic rules include such as BFU based on product, service modules, organization hierarchical structures and market regions etc. These selected rules must correspond to objective of the fractalbased SC. For example, manufacturing SC is based on product modules to construct BFU, one product module usually make of supplier, manufacture, retailer and reverse activities organizer, and the fractal-based SC also make up of parts such as supply, manufacture, sale, return and organization, the module and the SC have 'holography' characteristic, so constructing BFU can be in the light of the product modules. In practice, project departments in company usually reflect such idea. And then, the service SC is based on service projects to construct BFU. Secondly, 4PL is based on the selected fractal rules, and establishes the BFU similarity degree evaluating criteria set. 
The set is determined by the deferent fractal rules of the SC objective and sub-objective, established by 4PL together with members in fractal-based SC. For example, Shengbin Ni (2003) represented the criteria are involved organization structure, operation mode, organization culture and sharing platform together with 23 items. In practice, 4PL can design the basic criteria, and can revise them continually under the special rules, thus, some relative accurate, perfect and fine criteria can depict the similarity degree among BFUs. Lastly, based on the BFU data of criteria, using the self-similarity degree method together with the fractal character, we can determine BFUs in fractalbased SC. Although the traditional methods, such as fuzzy clustering technique, topological graph theory etc., those can measure the similarity degree of objects from different angles, but those are not suitable for depicting similarity degree of more than two BFUs. BFU structure and its characteristic determined need to introduce fractal dimension to depict similarity degree of more than two BFUs, usually it shall accord with the rule of constructing SC.

The fractal dimension can be used in scaling the complexity degree of objects, the proportion similarity degree criteria among $N$ evaluating criteria of any two $\operatorname{BFUs}(x, y)$ in $s$ similar hierarchical SC BFUs, whcih is regarded as the discrete distribution set in $N$ - dimension space, then the fractal dimension of the set can be used scale the discrete degree of these criteria in $\mathrm{N}$ dimension space. These criteria often include the quantitative and qualitative criteria. The quantitative criteria can be obtained by the enterprise's historical data directly, such as average turnover ratio, average manufacturing period etc. The qualitative criteria can be obtained by some experts evaluating value, such as customer satisfactory degree, employee devotion degree etc. Suppose there are $k$ experts to evaluate $l$ qualitative criteria via percentage, assume that their values belong to [0.100], higher value denotes smaller difference between two BFUs in this criteria. Let the evaluated results are as follows:

$$
m(x, y)=\left[\begin{array}{cccc}
m_{1,1} & \cdots & \cdots & m_{1, l} \\
m_{2,1} & \cdots & \cdots & m_{2, l} \\
\cdots & \cdots & m_{i, j} & \cdots \\
m_{k, 1} & \cdots & \cdots & m_{k, l}
\end{array}\right]_{k \times l} \quad \begin{aligned}
& i=1, \cdots, k, j=1, \cdots l, \\
& x=1, \cdots, s, y=1, \cdots, s
\end{aligned}
$$

Where $m_{i, j}$ is $i$ expert given evaluating value for $\operatorname{BFU} f_{x}, f_{y}$, and $j$ criteria.

Suppose that $\bar{m}(x, y)=\left(\bar{m}_{1}, \cdots, \bar{m}_{j}, \cdots, \overline{m_{l}}\right)$, where $\bar{m}_{j}=\frac{\sum_{i=1}^{k} m_{i, j}}{k}$, then we will normalize these data. Let

$$
z_{i}(x, y)=\left\{\begin{array}{l}
x_{i} / y_{i}, x_{i} \neq y_{i} \\
\bar{m}_{i} / 100, x_{i}=y_{i}
\end{array}\right.
$$


where, $x_{i}$ and $y_{i}$ are the relative quantitative criteria of two BFUs, respectively; $\overline{m_{i}}$ is average value of $i$ qualitative criteria by multiple comparison of BFUs. In this way, $\left.Z_{i}(x, y)\right\}_{i=1}^{N}$ makes up of set in $N$-dimension, $Z_{i}(x, y)$ denotes a point in $N$-dimension, here, metric is defined by Euclidean distance. Consider a ball has $r$ radius, let $R=\max _{i=1 \cdots N}\left[Z_{i}(x, y)\right]$. Assume that there is a point $M(r)$ in the ball, $M(r)=\sum_{i=1}^{N} H\left(r-z_{i}\right)$, where $H(x)$ is a Heaviside's function, i.e., $H(x)=\left\{\begin{array}{ll}1, & x>0 \\ 0, & x \leq 0\end{array}\right.$, when $r \in[0, R], M(r)$ is a power value about $r$, i.e., $M(r) \infty r^{D}$. Then, set $\left.Z_{i}(x, y)\right\}_{i=1}^{N}$ reflects the fractal characteristic, and its fractal dimension $D(x, y)$ satisfies:

$$
D(x, y)=\frac{\ln M(r)}{\ln r}
$$

To $\ln M(r)-\ln r$, using by linear regression technique, we can calculate the fractal dimension $D$. The similarity degree deviation is defined in absolute value between $D$ and $N$, namely

$$
\operatorname{dev}(x, y)=|D(x, y)-N|
$$

The value depicts the similarity degree of two BFUs, smaller value, shows the similarity degree of two BFUs is higher, whereas, the similarity degree of two BFUs is smaller. Therefore, we can obtain similarity degree deviation among $s(s-1) / 2$ identical SCs' BFUs. Using by the shortest distance to clustering, we can receive the highest BFU or the priority of BFU in a SC. Let $d(x, y)=\operatorname{dev}(x, y)$, then

$$
d^{2}(x, y)=\operatorname{dev}^{2}(x, y)=(D(x, y)-N)^{2}
$$

Based on recurrence formula, we have

$$
d^{2}(u, v)=\frac{1}{2} d^{2}(v, x)+\frac{1}{2} d^{2}(v, y)-\frac{1}{2}\left|d^{2}(v, x)-d^{2}(v, y)\right|
$$

where, BFU $f_{x}$ and $f_{y}$ merge into a new BFU $f_{u}, d^{2}(u, v)$ is square value of the distance between $\mathrm{BFU} f_{u}$ and any $\mathrm{BFU} f_{v}, d^{2}(v, x)$ or $d^{2}(v, y)$ is square value of the distance between $f_{x}$ and $f_{v}$ of $f_{y}$ and $f_{v}$, respectively. 


\section{Strategies}

When constructing the SC's BFU, 4PL depends on different kind of SCs, based on members information in $\mathrm{SC}$, it will adopt the different strategies; regulating and reengineering frequency is higher to the short-term SC, in order to achieve BFUs in SC momentarily, 4PL needs help the SC to select new members continually to substitute unsuited enterprises. In this case, 4PL needs set up a comprehensive enterprise database, to make for it regulate structure and renew members in a SC effectively, at the same time, 4PL needs often update the database. A perfect database is easy to 4PL develop its operation in future. With reengineering frequency of the medium-term SC reducing, 4PL just needs regulate parts of all levels BFUs in a SC, and achieve BFUs optimization. In this case, 4PL must further consummate the database to regulate BFUs. The regulating and reengineering frequency is very smallest to the long-term SC, so 4PL just regulates BFUs in lesser scale to realize BFUs optimization in succession. Herein, 4PL should stress to mine information of existing in a SC's members, so support decision-making that improving the SC operation.

\section{Plan and implementation}

4PL shall divide SC into some BFUs, depend on integrated objective and all BFUs subobjective, and combine with BFUs activities to determine plan, for example, production plan and service plan in BFUs etc. These plans need reach coherence among 4PL together with members of a SC, thus 4PL together with the members of SC implement the corresponding plan. In implementing process, 4PL should attach its key ability and advantage to finish the coordinating tasks of a SC. Since all BFUs have the intrinsic characteristics, such as self-organization and selfoptimization, BFUs will make use of those characteristics to implement the coordination and operation of a SC to the non-specialization fields of 4PL. 4PL can use the nesting rules of BFUs, to control the coordination of higher hierarchy SC; To the specialization fields of 4PL, 4PL can participate in the coordination and operation of SC, availably improve the coordination of the SC continually.

\section{Performance evaluation and feedback control}

To Coordinate the activity plan of SC and gain effect by 4PL, we often need compare with objective of SC, so utilizing the SC's performance evaluation and control activities has more significance. 4PL needs together with the members of SC to set up the performance evaluation system for all kinds of BFUs, so as to achieve the real time feedback control in the dynamic environment. When some criteria of BFU can not reach, then we will review the BFU are whether or not in BFU's space, i.e., it is whether in the three-dimension space built by objective, resource and restriction. If the BFU still is in the space, there exist two approaches to achieve

their optimization: (1) Formed enterprises of the BFU utilize the self-optimization rule to implement self-optimizing process in BFU, which can improve performance; (2) By using 4PL 
participation to implementing optimizing process, 4PL should analyze BFU's operating process and structure to reengineering $\mathrm{BFU}$, then can optimize the $\mathrm{BFU}$ and obtain the performance objective. If one BFU of SC is not in the BFU's activity space, there exist also two approaches to achieve BFU's optimization. One needs change the space, i.e., re-defined or programmed the BFU's objective, resource and restriction, then achieve its optimization; another don't change the space, by using 4PL participating and reengineering process of the BFU, in this way achieve its optimization. In a word, 4PL achieves the real time feedback control objective in the dynamic environment of SC.

As far as performance evaluation and feedback control to BFUs, 4PL needs depend on the different types of SCs, utilizes the different integrated performance criteria systems. From whole society, the waiting selection enterprises are largest to the short-term SC, 4PL may be confronted with enterprises of multi-industry, those enterprises are much diversity, and the SC's life cycle is shorter, 4PL joined in the SC time may be shorter, and undertakes tasks are often complicated, therefore, 4PL should apply the integrated performance evaluating system, then can be in favor of constructing a SC or selecting enterprises rapidly, such as SCOR or ISO. The waiting selection enterprises are still largest to the medium-term SC, 4PL may be faced with enterprises of multiindustry, those enterprises are much diversity too, 4PL can apply the integrated performance evaluating system of SC or enterprise to construct SC or select enterprises as quickly as possible. However, with the cooperative period extending in a SC, the integrated performance evaluating system can evaluate the SC or enterprise exactly,. The members in a long-term SC are quite changeless, and the SC's long-term strategy is an important work for 4PL, by using the specialization customized performance evaluating system to evaluate SC or enterprises, that is optimum choice.

\section{Disorganization}

When the SC's BFUs or the whole SC go through the entire life cycle, they must be up against disorganization. Under this case, 4PL needs dispose the relative affairs, such as how to establish the enterprises' existing mechanism; 4PL does his possible to lower the disadvantage for BFU's disorganization, such as how to innovate management properly due to SC abruption resulted in operating ceasing. From control scale of the entire SC coordination, 4PL coordinates BFU system can be divided into three levels (described as Figure 7).

First level is self-optimization of BFU, 4PL mainly makes use of characteristics of selforganization and self-optimization in this level, realizes the local self-optimization in a SC; second level is the structural optimization of BFU, 4PL needs pay attention to the regulating and reengineering of BFU's structure and members in the level; third level is the strategic optimization, 4PL's main tasks include regulation of strategic objective, re-defined and division of resource and restriction in a SC in this level. In a general way, 4PL usually participates in the SC's coordination in second and third level, and is concerned with the SC's coordination in first level indirectly. 


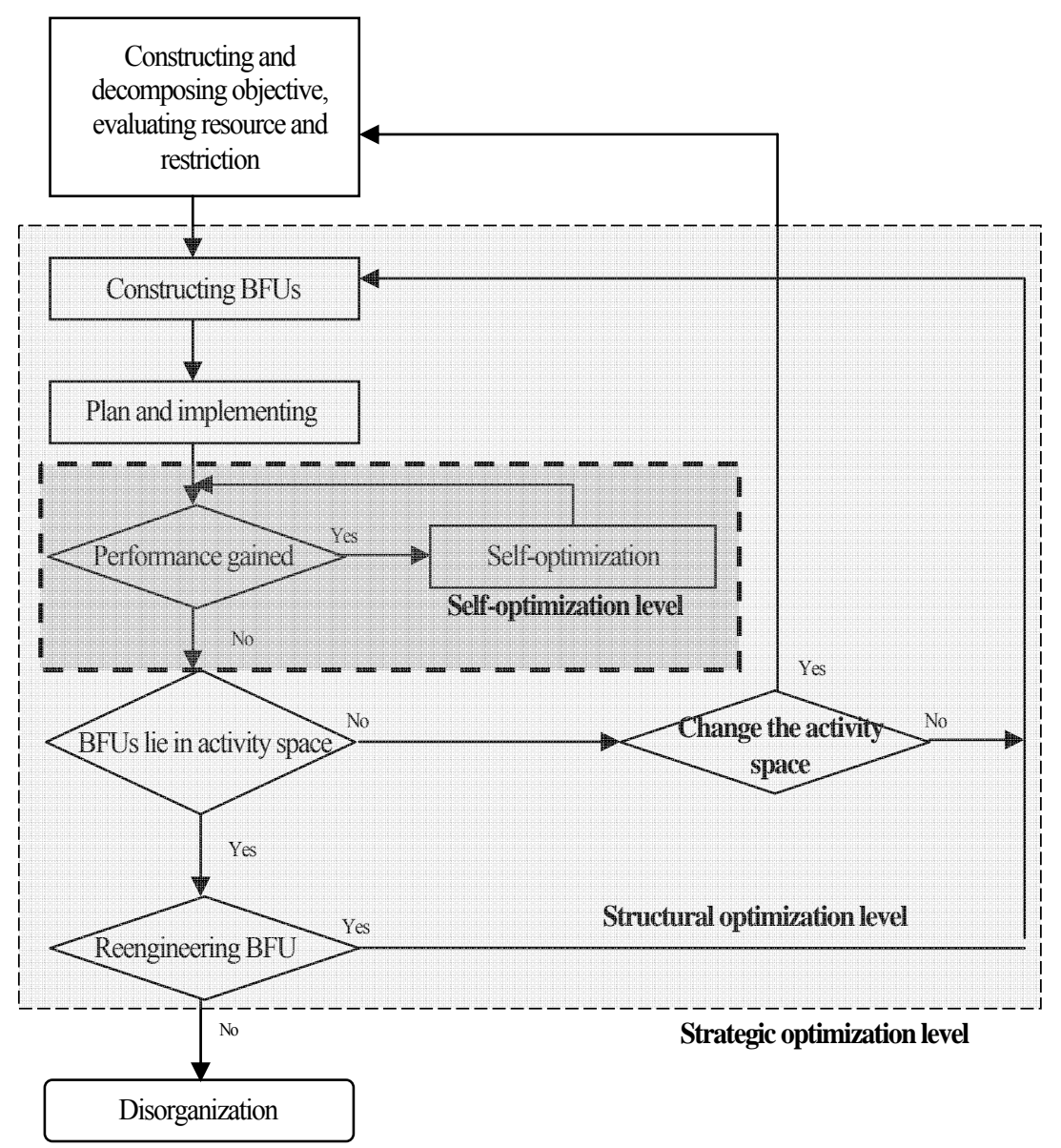

Figure 7. 4PL coordinates BFU's system 


\section{Case study}

Alcatel-Lucent Technologies, Inc. is a global leader provider in telecom equipment field, manufactures products used to build communications network infrastructure. Its copper line transmission and switching, wireless, and optical gear is used in core telephony and data networks worldwide. The company also makes communications and network management software and provides a wide range of services. Many of Alcatel-Lucent's products are developed by its Bell Laboratories research and development unit. The company provides wireline and wireless products to leading telephone companies and other communications service providers. Since 1990s, with its product demand blooming, to press close to customer, Alcatel-Lucent Technologies, Inc. ceaselessly extends its storage and service network. Up to 1998, Alcatel-Lucent Technologies, Inc. has 240 distributor centers spread over America region, herein results in the relative cost increases rapidly. When Lucent Technologies, Inc. is up against income gain rate decreasing and how to reduce cost, it began implementing the outsourcing strategy of manufacture and logistics. From 1999, AlcatelLucent Technologies, Inc. first built leading logistics provider (LLP) relationship, through Ryder ran Alcatel-Lucent's supply chain across regions involving North America, Caribbean and Latin America. LLP is as the primary phase of 4PL., its objective is that improve the efficiency of supply chain and the distributing facilities, decrease touch point and turnover time. Ryder helps AlcatelLucent implement 4PL service process as follows:

1. Set up team: When the outsourcing agreement subscribed, the relative personnel (such as transportation experts, industrial Engineering experts, network designers) of Ryder buildup a team promptly enter into Alcatel-Lucent Technologies, Inc., and launch on improving Alcatel-Lucent's complicated storage and the distributing facilities.

2. Put forward plan and actualize: By Ryder's personnel work with the designers and network engineers of Alcatel-Lucent, 240 distributing points decrease to 14 in 2000. One of measures adopted by Ryder is constructing a Logistics Service Centers (LSC) network, those centers supply parts, telecom exchange facilities and other materials to Alcatel-Lucent and its customers. Under Ryder's advice, two key suppliers (Anixter and Graybar) could retain the enough inventory in all LSC. Such regional cooperative strategy results in the delivery process become more flatness.

3. Continual improve: Ryder together with Alcatel-Lucent constituted Key Performance Indicator (KPI) and Balance Score Card to monitor everyday supply chain operational efficiency. They together determine their objective, and continual improved based on timing frame and other factors. At present, Ryder has take over the whole logistics operation aim at the American market, involving in network design and implement, negotiate, transport plan and administer, return management (reverse logistics activity), logistics service bill and audit etc. Moreover, Alcatel-Lucent still built relationship with UPS Global Logistics to optimize the supply chain in region of Europe, Middle East, Asia and the Pacific and China.

During 4PL managing Alcatel-Lucent's supply chain process, Alcatel-Lucent took action as follows:

1. Evaluate 4PL's core ability when handing over the logistics activities: Alcatel-Lucent 
established a comprehensive plan for the supply chain operation handing over 4PL. The entire process began from warehouse, passed through half in two years. Since AlcatelLucent's manager found Ryder and UPS have stronger warehouse management ability. From beginning, Alcatel-Lucent's manager selected an experimental market to test 4PL mode in order to ensure the process reliability and information unblocked. Even at present, Alcatel-Lucent still continues to evaluate the supply chain and the logistics process constructed by 4PL.

2. Use the region logistics and the distributing team to manage 4PL. Alcatel-Lucent set up the region logistics and the distributing team to manage LLP. Every region team directly takes charge transport management, warehouse management, IT support and integration, and forward or reverse logistics management. When there needs adequate customization techniques at same time to satisfy many region demand of the supply chain, the region team can apply the Global Standard Practices to improve Alcatel-Lucent's operation efficiency.

3. Emend each objective to improve 4PL performance: Alcatel-Lucent adopts the following measures such that 4PL's objective accord with Alcatel-Lucent's objective: Perform the Payfor-Performance mechanism. The mechanism not only inspires 4PL to improve efficiency and reduce cost, but also make 4PL gain award while excess expect indicator, or else obtain chastisement; Set up the service level agreement. Alcatel-Lucent together with 4PL sets up a Service Level Agreements (SLAs) to monitor the operation that affect performance activities; Supervise neutralism of the leading logistics. To avoid 4PL to use its preference conveyancer or warehouseman, Lucent has a supplier management team to supervise neutralism of 4PL.

Alcatel-Lucent uses 4PL's core ability and capacity to construct its supply chain and gain higher profit, results in Lucent concentrate in research and development and does not fall into the daily affairs of its supply chain. Table 1 illustrates the fore-and-aft indexes change of implementing 4PL for Alcatel-Lucent in China. From April 2002 to January 2003, the global transport costs economize \$7000,000 for Alcatel-Lucent in China.

From the case, the following discussions are based on the abovementioned conclusions:

1. From the supply chain types: Before 4PL entered, Alcatel-Lucent already has a comparatively self-contained supply chain management system, the supply chain type is a centralized supply chain. When 4PL entered, Ryder reengineered Alcatel-Lucent's distributing network, such that 240 distributing point integrated 14 in American market. In fact, the relationship between Alcatel-Lucent and 4PL is 'symbiont', so it acts in accord with the coordinating strategies of long-term or medium-term supply chain.

2. From the transaction cost analysis: Alcatel-Lucent handed over the supply chain operation is ongoing gradually, firstly it handed over the warehouse management, found the effect after 4PL introduced, then set over the entire supply chain operation to 4PL. Based on the transaction cost models of abovementioned conclusions, 4PL always begin from the economizing transaction cost operational activities, extends the coordinating tasks to the whole supply chain. For example, Ryder has reengineered the distributing network for Alcatel-Lucent, increased the distributing concentration degree, decreased the touch points, 
lowered the complex degree in the early supply chain, then it can reduce the transaction cost happened in Alcatel-Lucent and forwarder. The relationship between Alcatel-Lucent and $4 \mathrm{PL}$ is 'symbiont', results in the opportunism behavior lowering, thus decreases the transaction cost appeared in two 4PLs coordinating Alcatel-Lucent's supply chain.

Table 1

The fore-and-aft indexes change of implementing 4PL for Alcatel-Lucent in China

\begin{tabular}{|c|c|c|}
\hline & Before 4PL entered & After 4PL entered \\
\hline Logistics suppliers & 19 & one $4 \mathrm{PL}$ \\
\hline Warehouse amount & $\begin{array}{c}5 \text { region warehouses, } 36 \text { project } \\
\text { warehouses }\end{array}$ & $\begin{array}{c}4 \text { region warehouses, } 13 \text { project } \\
\text { warehouses }\end{array}$ \\
\hline Average logistics period & 14 & 12 \\
\hline Timely delivery rate timely & $80 \%$ & $91 \%$ \\
\hline $\begin{array}{c}\text { Logistics management } \\
\text { department }\end{array}$ & $\begin{array}{l}\text { One in } 13 \text { corporation units, } \\
\text { respectively }\end{array}$ & Just one \\
\hline Average order delivery times & 6 & 3 \\
\hline $\begin{array}{l}\text { For example: distributing process } \\
\text { for CDMA product in China }\end{array}$ & Warehouse utilizing rate: $61 \%$ & $\begin{array}{l}\text { Warehouse utilizing rate: } 72 \% \text {, and } \\
\text { Warehouse amount decreasing } 64 \%\end{array}$ \\
\hline
\end{tabular}

In a word, after 4PL entered, Alcatel-Lucent's supply chain operation receives remarkable effect, some key performance indicators are improved. Certainly, 4PL has started its journey, many coordinating tasks wait for 4PL in future.

Alcatel-Lucent uses 4PL's core ability and capacity to construct its SC and gain higher profit, results in Lucent concentrate in research and development and does not fall into the daily affairs of its SC. From April 2002 to January 2003, the global transport costs economize $\$ 7000,000$ for Alcatel-Lucent in China. Now, we consider the SC typically selected from Alcatel-Lucent in China, where a $4 \mathrm{PL}$ needs use 5 primary BFUs A, B, C, D and E in SC to construct coordination BFU. Now, we review the similarity degree of 5 primary BFUs, and integrate BFUs in fractal-based SC to form coordination BFU. The criteria and their variables of the similarity degree of BFUs are described in Table 2. The quantitative criteria of 5 primary BFUs are reflected in Table 3. The qualitative criteria have been from experts evaluated, e.g., those experts come from such as professional CEO and professors, the qualitative criteria of BFU A and BFU B are represented in Table 4. The data collected were based on Alcatel-Lucent in China, so these are reliability and validity. Using by normalization formula, the criteria values are described in Table 5 . 
Table 2

The criteria and their expressed variables of the similarity degree of BFUs

\begin{tabular}{|c|c|c|c|c|c|}
\hline \multicolumn{4}{|c|}{ Qualitative criteria } & \multicolumn{2}{|c|}{ Quantitative criteria } \\
\hline Content & Variable & Content & Variable & Content & Variable \\
\hline $\begin{array}{c}\text { Employee } \\
\text { satisfactory degree }\end{array}$ & $\mathrm{C} 1$ & $\begin{array}{l}\text { Logistics sites adjacent } \\
\text { degree }\end{array}$ & C9 & $\begin{array}{l}\text { Average educated years } \\
\text { for employee }\end{array}$ & $\mathrm{C} 17$ \\
\hline $\begin{array}{c}\text { Employee } \\
\text { devotion degree }\end{array}$ & $\mathrm{C} 2$ & $\begin{array}{l}\text { Production resources } \\
\text { sharing degree }\end{array}$ & $\mathrm{C} 10$ & $\begin{array}{c}\text { Average order processing } \\
\text { time }\end{array}$ & $\mathrm{C} 18$ \\
\hline $\begin{array}{c}\text { Recognizable } \\
\text { degree to } \\
\text { organization value }\end{array}$ & C3 & $\begin{array}{c}\text { Employee professional skill } \\
\text { adjacent degree }\end{array}$ & C11 & $\begin{array}{l}\text { Average manufacturing } \\
\text { period }\end{array}$ & $\mathrm{C} 19$ \\
\hline $\begin{array}{c}\text { Team spirit } \\
\text { adjacent degree }\end{array}$ & $\mathrm{C} 4$ & $\begin{array}{c}\text { Administrative organization } \\
\text { complexity degree }\end{array}$ & $\mathrm{C} 12$ & Average distribution time & $\mathrm{C} 20$ \\
\hline $\begin{array}{l}\text { Innovation ability } \\
\text { adjacent degree }\end{array}$ & $\mathrm{C} 5$ & $\begin{array}{l}\text { Administrative structure } \\
\text { regularity degree }\end{array}$ & $\mathrm{C} 13$ & $\begin{array}{l}\text { Average inventory } \\
\text { turnover }\end{array}$ & $\mathrm{C} 21$ \\
\hline $\begin{array}{l}\text { Technology } \\
\text { sharing degree }\end{array}$ & C6 & $\begin{array}{l}\text { Administrative structure } \\
\text { centralized power degree }\end{array}$ & $\mathrm{C} 14$ & $\begin{array}{l}\text { Average emergency } \\
\text { feedback time }\end{array}$ & $\mathrm{C} 22$ \\
\hline $\begin{array}{c}\text { Human-resource } \\
\text { platform sharing } \\
\text { degree } \\
\text { Information } \\
\text { sharing degree }\end{array}$ & $\mathrm{C} 8$ & $\begin{array}{l}\text { Administrative structure } \\
\text { scale } \\
\text { Product relevant degree }\end{array}$ & $\mathrm{C} 16$ & $\begin{array}{c}\text { 'Pull' management } \\
\text { proportion in inventory } \\
\text { management }\end{array}$ & $\mathrm{C} 23$ \\
\hline
\end{tabular}

Table 3

The quantitative criteria of 5 primary BFUs

\begin{tabular}{l|c|c|c|c|c|c}
\hline Quantitative criteria & BFU A & BFU B & BFU C & \multicolumn{2}{l|}{ BFU D } & \multicolumn{2}{l|}{ BFU E } \\
\hline \hline Average educated years for employee (year) & $\mathrm{C} 17$ & 15.2 & 15.3 & 15.1 & 15 & 15.4 \\
Average order processing time (hour) & $\mathrm{C} 18$ & 3.32 & 3.36 & 3.34 & 3.37 & 3.31 \\
Average manufacturing time (hour) & $\mathrm{C} 19$ & 15.2 & 15.3 & 15.2 & 15.4 & 15.1 \\
Average distribution (day) & $\mathrm{C} 20$ & 3.23 & 3.22 & 3.25 & 3.24 & 3.23 \\
Average inventory turnover & $\mathrm{C} 21$ & 1.56 & 1.55 & 1.53 & 1.54 & 1.52 \\
Average emergency feedback time (hour) & $\mathrm{C} 22$ & 0.32 & 0.33 & 0.33 & 0.32 & 0.31 \\
'Pull' management proportion in inventory management & $\mathrm{C} 23$ & 0.67 & 0.66 & 0.68 & 0.69 & 0.65 \\
\hline
\end{tabular}


Study on 4PL as coordinating and constructing agent for supply chain systems:

transaction cost theory approach and wave-particle duality

Table 4

The qualitative criteria of BFU A and BFU B obtained by experts evaluated

\begin{tabular}{c|c|c|c|c|c|c|c|c|c|c|c|c|c|c|c|c}
\hline $\begin{array}{c}\text { Criteria } \\
\text { variable }\end{array}$ & $\mathrm{C} 1$ & $\mathrm{C} 2$ & $\mathrm{C} 3$ & $\mathrm{C} 4$ & $\mathrm{C} 5$ & $\mathrm{C} 6$ & $\mathrm{C} 7$ & $\mathrm{C} 8$ & $\mathrm{C} 9$ & $\mathrm{C} 10$ & $\mathrm{C} 11$ & $\mathrm{C} 12$ & $\mathrm{C} 13$ & $\mathrm{C} 14$ & $\mathrm{C15}$ & $\mathrm{C} 16$ \\
\hline \hline & 88 & 97 & 91 & 99 & 91 & 94 & 88 & 97 & 94 & 86 & 95 & 90 & 88 & 98 & 98 & 97 \\
5 experts score $m(A, B)$ & 93 & 90 & 96 & 92 & 93 & 98 & 99 & 92 & 91 & 85 & 87 & 89 & 97 & 95 & 90 & 89 \\
& 96 & 93 & 97 & 94 & 86 & 96 & 85 & 86 & 95 & 91 & 96 & 93 & 92 & 91 & 91 & 87 \\
& 98 & 91 & 92 & 97 & 99 & 98 & 93 & 85 & 89 & 86 & 88 & 97 & 98 & 94 & 86 & 91 \\
& 86 & 98 & 95 & 85 & 87 & 88 & 90 & 94 & 90 & 99 & 85 & 96 & 97 & 87 & 85 & 95 \\
\hline $\bar{m}(A, B)$ & 0.92 & 0.94 & 0.94 & 0.93 & 0.91 & 0.95 & 0.91 & 0.91 & 0.92 & 0.89 & 0.9 & 0.93 & 0.94 & 0.93 & 0.9 & 0.92 \\
\hline
\end{tabular}

Table 5

The criteria values by using normalization

\begin{tabular}{c|c|c|c|c|c|c|c|c|c|c}
\hline $\begin{array}{c}\text { Criteria } \\
\text { variable }\end{array}$ & $z(\mathrm{~A}, \mathrm{~B})$ & $z(\mathrm{~A}, \mathrm{C})$ & $z(\mathrm{~A}, \mathrm{D})$ & $z(\mathrm{~A}, \mathrm{E})$ & $z(\mathrm{~B}, \mathrm{C})$ & $z(\mathrm{~B}, \mathrm{D})$ & $z(\mathrm{~B}, \mathrm{E})$ & $z(\mathrm{C}, \mathrm{D})$ & $z(\mathrm{C}, \mathrm{E})$ & $z(\mathrm{D}, \mathrm{E})$ \\
\hline \hline $\mathrm{C} 1$ & 0.922 & 0.940 & 0.904 & 0.936 & 0.910 & 0.900 & 0.958 & 0.924 & 0.920 & 0.938 \\
$\mathrm{C} 2$ & 0.938 & 0.888 & 0.902 & 0.940 & 0.896 & 0.934 & 0.930 & 0.890 & 0.934 & 0.908 \\
$\mathrm{C} 3$ & 0.942 & 0.932 & 0.886 & 0.924 & 0.946 & 0.928 & 0.914 & 0.920 & 0.898 & 0.924 \\
$\mathrm{C} 4$ & 0.934 & 0.940 & 0.900 & 0.942 & 0.940 & 0.958 & 0.898 & 0.888 & 0.906 & 0.938 \\
$\mathrm{C} 5$ & 0.912 & 0.920 & 0.916 & 0.946 & 0.894 & 0.928 & 0.926 & 0.928 & 0.896 & 0.898 \\
$\mathrm{C} 6$ & 0.948 & 0.900 & 0.902 & 0.930 & 0.906 & 0.894 & 0.920 & 0.924 & 0.876 & 0.908 \\
$\mathrm{C} 7$ & 0.910 & 0.936 & 0.928 & 0.934 & 0.890 & 0.886 & 0.912 & 0.936 & 0.938 & 0.868 \\
$\mathrm{C} 8$ & 0.908 & 0.912 & 0.942 & 0.912 & 0.940 & 0.920 & 0.896 & 0.930 & 0.888 & 0.920 \\
$\mathrm{C} 9$ & 0.918 & 0.936 & 0.902 & 0.930 & 0.912 & 0.944 & 0.940 & 0.938 & 0.904 & 0.916 \\
$\mathrm{C} 10$ & 0.894 & 0.926 & 0.916 & 0.942 & 0.888 & 0.892 & 0.928 & 0.912 & 0.894 & 0.928 \\
$\mathrm{C} 11$ & 0.902 & 0.918 & 0.938 & 0.904 & 0.890 & 0.940 & 0.948 & 0.916 & 0.916 & 0.914 \\
$\mathrm{C} 12$ & 0.930 & 0.908 & 0.936 & 0.920 & 0.922 & 0.922 & 0.912 & 0.910 & 0.918 & 0.920 \\
$\mathrm{C} 13$ & 0.944 & 0.886 & 0.928 & 0.930 & 0.950 & 0.906 & 0.914 & 0.896 & 0.924 & 0.902 \\
$\mathrm{C} 14$ & 0.930 & 0.926 & 0.938 & 0.926 & 0.906 & 0.922 & 0.912 & 0.916 & 0.920 & 0.890 \\
$\mathrm{C} 15$ & 0.900 & 0.928 & 0.928 & 0.934 & 0.916 & 0.892 & 0.956 & 0.934 & 0.876 & 0.916 \\
$\mathrm{C} 16$ & 0.918 & 0.880 & 0.930 & 0.894 & 0.918 & 0.916 & 0.912 & 0.918 & 0.922 & 0.954 \\
$\mathrm{C} 17$ & 0.993 & 1.007 & 1.013 & 0.987 & 1.013 & 1.020 & 0.994 & 1.007 & 0.981 & 0.974 \\
$\mathrm{C} 18$ & 0.988 & 0.994 & 0.985 & 1.003 & 1.006 & 0.997 & 1.015 & 0.991 & 1.009 & 1.018 \\
$\mathrm{C} 19$ & 0.993 & 1.000 & 0.987 & 1.007 & 1.007 & 0.994 & 1.013 & 0.987 & 1.007 & 1.020 \\
$\mathrm{C} 20$ & 1.003 & 0.994 & 0.997 & 1.000 & 0.991 & 0.994 & 0.997 & 1.003 & 1.006 & 1.003 \\
$\mathrm{C} 21$ & 1.006 & 1.020 & 1.013 & 1.026 & 1.013 & 1.006 & 1.020 & 0.994 & 1.007 & 1.013 \\
$\mathrm{C} 22$ & 0.970 & 0.970 & 1.000 & 1.032 & 1.000 & 1.031 & 1.065 & 1.031 & 1.065 & 1.032 \\
$\mathrm{C} 23$ & 1.015 & 0.985 & 0.971 & 1.031 & 0.971 & 0.957 & 1.015 & 0.986 & 1.046 & 1.062 \\
\hline$R$ & 1.015 & 1.020 & 1.013 & 1.032 & 1.013 & 1.031 & 1.065 & 1.031 & 1.065 & 1.062 \\
\hline
\end{tabular}


To $\ln r-\ln M(r)$, using by linear regression technique, we can calculate the fitted fractal dimension $D$, Figure 8 illustrates all calculated results. The similarity degree deviation $\mathrm{dev}$ and $d^{2}$ are shown in Table 6 . The clustering analysis results are represented in Table 6 , the clustered treemap is illustrated in Figure 9. By using the clustering analysis, we can find BFU A and BFU E are most similarity, so integrating them to BFU AE, in this way, through the first time integrated, we will construct 4 BFUs (AE, B, C, D); Among 4 BFUs, BFU AE and B are most similarity, then integrating them to $\mathrm{BFU} A E B$, by second time integrated, we will construct 3 BFUs (AEB, C, D); Among 3 BFUs, $\mathrm{AEB}$ and $\mathrm{C}$ are most similarity, then integrating them to $\mathrm{AEBC}$, by third time integrated, we can construct 2 BFUs (AEBC, D). Therefore, using by the clustering analysis, we can receive the highest $\mathrm{BFU}$ or the priority of $5 \mathrm{BFUs}$ in the $\mathrm{SC}$.
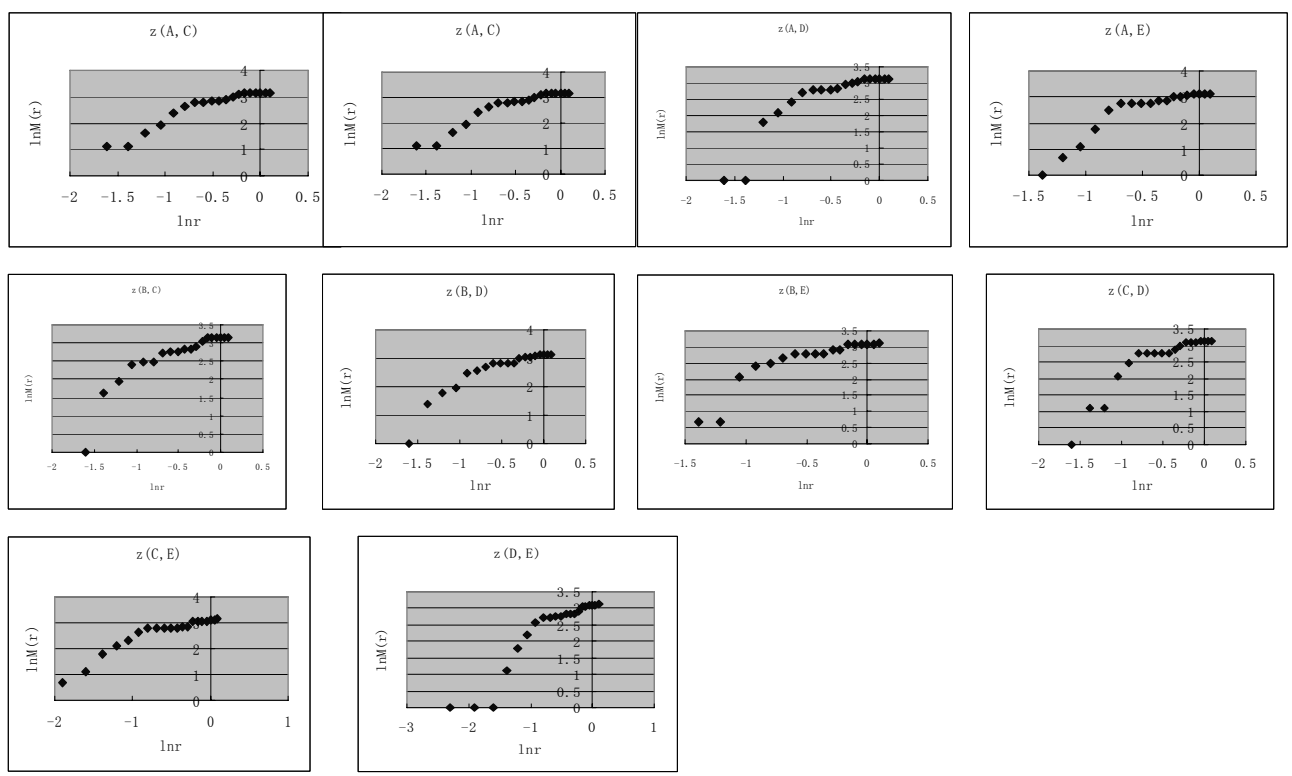

Figure 8. Double-logarithm map of $\ln r-\ln M \quad(r)$ 


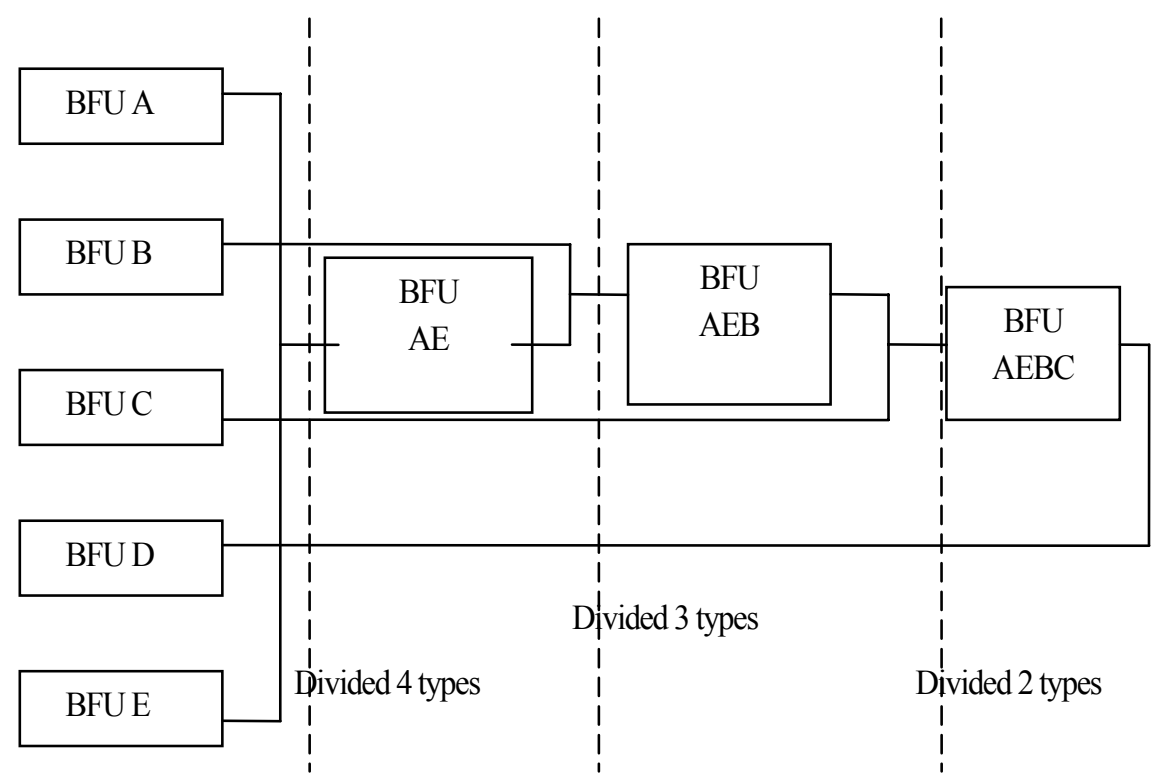

Figure 9. BFUs A, B, C, D, E clustering tree map

Table 6

Fractal-dimension $D$, similarity degree deviation $\mathrm{dev}$ and $d^{2}$ calculating results

\begin{tabular}{l|l|c|c}
\hline \multicolumn{2}{c|}{ Fractal dimension $D$} & Similarity degree deviation $d e v=|D-N|, N=23$ & $d^{2}=d e v^{2}$ \\
\hline \hline$D(\mathrm{~A}, \mathrm{~B})$ & 1.472491 & 21.52751 & 463.4336 \\
$D(\mathrm{~A}, \mathrm{C})$ & 1.559742 & 21.44026 & 459.6847 \\
$D(\mathrm{~A}, \mathrm{D})$ & 1.165024 & 21.83498 & 476.7662 \\
$D(\mathrm{~A}, \mathrm{E})$ & 1.955769 & 21.04423 & 442.8597 \\
$D(\mathrm{~B}, \mathrm{C})$ & 1.066606 & 21.93339 & 481.0738 \\
$D(\mathrm{~B}, \mathrm{D})$ & 1.247105 & 21.75290 & 473.1885 \\
$D(\mathrm{~B}, \mathrm{E})$ & 1.889916 & 21.11008 & 445.6356 \\
$D(\mathrm{C}, \mathrm{D})$ & 1.513878 & 21.48612 & 461.6534 \\
$D(\mathrm{C}, \mathrm{E})$ & 1.125065 & 21.87494 & 478.5128 \\
$D(\mathrm{D}, \mathrm{E})$ & 1.085163 & 21.91484 & 480.2601 \\
\hline
\end{tabular}


Table 6

BFUs A, B, C, D, E clustering calculating results

\begin{tabular}{|c|c|c|c|c|c|}
\hline \multicolumn{2}{|c|}{ First time integrated } & \multicolumn{2}{|c|}{ Second time integrated } & \multicolumn{2}{|c|}{ Third time integrated } \\
\hline$d^{2}(\mathrm{~A}, \mathrm{~B})$ & 463.4336 & $d^{2}(\mathrm{AE}, \mathrm{B})$ & $\underline{445.6356}$ & $d^{2}(\mathrm{AEB}, \mathrm{C})$ & $\underline{459.6847}$ \\
\hline$d^{2}(\mathrm{~A}, \mathrm{C})$ & 459.6847 & $d^{2}(\mathrm{AE}, \mathrm{C})$ & 459.6847 & $d^{2}(\mathrm{AEB}, \mathrm{D})$ & 473.1885 \\
\hline$d^{2}(\mathrm{~A}, \mathrm{D})$ & 476.7662 & $\left.d^{2} \mathrm{AE}, \mathrm{D}\right)$ & 476.7662 & $d^{2}(\mathrm{C}, \mathrm{D})$ & 461.6534 \\
\hline$d^{2}(\mathrm{~A}, \mathrm{E})$ & $\underline{442.8597}$ & $d^{2}(B, C)$ & 481.0738 & & \\
\hline$d^{2}(B, C)$ & 481.0738 & $d^{2}(\mathrm{~B}, \mathrm{D})$ & 473.1885 & & \\
\hline$d^{2}(\mathrm{~B}, \mathrm{D})$ & 473.1885 & $d^{2}(\mathrm{C}, \mathrm{D})$ & 461.6534 & & \\
\hline$d^{2}(\mathrm{~B}, \mathrm{E})$ & 445.6356 & & & & \\
\hline$d^{2}(\mathrm{C}, \mathrm{D})$ & 461.6534 & & & & \\
\hline$d^{2}(\mathrm{C}, \mathrm{E})$ & 478.5128 & & & & \\
\hline$d^{2}(\mathrm{D}, \mathrm{E})$ & 480.2601 & & & & \\
\hline \multicolumn{2}{|c|}{$\begin{array}{l}\text { Least value } 442.8597 ; \mathrm{A}, \mathrm{E} \\
\text { integrated } \mathrm{AE}\end{array}$} & \multicolumn{2}{|c|}{$\begin{array}{l}\text { Least value } 445.6356 ; \mathrm{AE}, \mathrm{B} \\
\text { integrated } \mathrm{AEB}\end{array}$} & \multicolumn{2}{|c|}{$\begin{array}{l}\text { Least value } 459.6847 \text {; } \mathrm{AEB}, \mathrm{C} \text { integrated } \\
\mathrm{AEBC}\end{array}$} \\
\hline
\end{tabular}

\section{Conclusions}

With 4PLs are integrated into SCs, the selection of supply chains is an important issue as core competency of each enterprise must be matched prior to jointly implementing a unify business strategy/model. The prominent effect and function for $4 \mathrm{PL}$ is to integrate the $\mathrm{SC}$, which is typically beyond two stages, i.e. multi-stage modes. This paper introduces new fractal view to justify the concept of using 4PL, which is a contractor of SC coordination based on the division of community and the outsourcing development. The SC is the special organization whose characteristic has wave-particle duality. The proposed mathematical models enrich the connotation of SC and broaden the thought for 4PL development. Secondly, the proposed mathematical models based on transaction cost theory and fractal method, can be used as the theoretical analysis tool of 4PL coordinating supply chain evaluation. Finally, a case illustrated our conclusions.

\section{Acknowledgment}

This work is supported by new century outstanding talent plan in Fujian, China. 


\section{References}

Bhatnagar, R., Chandra, P. and Goyal, S. K. 1993. Models for multi-plant coordination. European Journal of Operation Research. 67: 141-160.

Bumstead, J. and Cannons, K. 2002. From 4PL to managed supply-chain operations. Focus Magazine. 5: 35-49.

Chopra, S. and Meindl, P. 2001. Supply chain management-strategy. Planning and Operation. USA: Prentice Hall, Inc. Press.

Cruz, M. 2000. Take it back: reverse supply chain companies meet demand. Computer Reseller News. 5(897): 3.

Deshmukh, A. V, Talavage, J. J. and Barash, M. M. 1998. Complexity in manufacturing systems, part 1: analysis of static complexity. IIE Transactions. 30:645-655.

Frizelle, G. and Woodcock, E. 1995. Measurement of complexity as an aid to developing operational strategy. International Journal of Operation and Production Management. 15(5):26-29.

Gattorna, J. and Selen, W. 2004. Characteristics, strategies and trends for 3PL/4PL in Australia. Alpha Research Consortium Research Report 2004.

Grover, V. and Malhotra, M. K. 2003. Transaction cost framework in operations and supply chain management research: theory and measurement. Journal of Operations Management. 21: 457-473.

Guide, V. D. R. and Van Wassenhove, L. N. 2003. Business aspects of closed-loop supply Chains. In: Guide, V.D.R. and Van Wassenhove, L.N. eds. Business aspects of closed-loop supply chains: exploring the issues. Pittsburgh, Pennsylvania. Carnegie Bosch Institute. 17-42.

Heok, Chong. 2001. Epilogue: UPS logistics-practical approaches to the e-supply chain. International Journal of Physical Distribution and Logistics Management. 6: 463-468.

Hobbs, Jill E. 1996. Transaction cost approach to supply chain management. Supply Chain Management. 1: 15-38.

Hong Hu et al. 2005. The studying on the relation between entropy and supply chain management system. Journal of Harbin University of Commerce. 6: 40-44.

http://www.alcatel-lucent.com/wps/portal.

Ji, G-J. 2006. Logistics and matter-element models based on firm innovative supply chains. International Journal of Operational Research. 2(1): 281-301.

Ji, G-J. 2006. Market-motivated value systems, reverse logistics and the evaluation model for the third party reverse logistics providers. Journal of International Logistics and Trade. 4 (1): 53-92.

Ji, G-J. 2008. Complaint management and virtual enterprise in closed-loop supply chains and its performance evaluation by using exergoeconomics and extenics. International Journal of Service and Operation Management. 2(2): 23-44. 
Krikke, H. R. et al. 2003. Concurrent product and closed-loop supply chain design with an application to refrigerators. International Journal of Production Research. 41(16): 3689-3719.

Martinez, J.I. and Jarillo, J.C. 1989. The evolution of research on coordination mechanisms in multinational research. Journal of International Business Studies. 3: 489-514.

Mintzberg, H. 1979. The structuring of organizations. Englewood Cliffs, NJ: Prentice-Hall.

Morrell, A. L. 2001. The forgotten child to the supply chain. Modern Materials Handling. 56(6): 33-36.

Ni, S.B., Chen, J.F., and Zhang, M. 2003. Self-similarity in fractal supply chain and its evaluating model. Management Science. 1(11): 46-52.

Panayides, P. M. and So, M. 2005. Logistics service provider-client relationships. Transportation Research. 41: 179-200.

Ryu, K., Shin, M., Kim, K., and Min, J. 2000. Intelligent control architecture for fractal manufacturing system. Proceedings of 3rd Asia-Pacific Conference on Industrial Engineering and Management Systems. Hong Kong. 594-598.

Ryu, K., Son, Y. and Min, J. 2002. Fractal-based supply chain management. Proceedings of 7th Annual International Conference. Busan, Korea. 24-26.

Sharma, M.K. and Bhagwat, R. 2006. Performance measurements in the implementation of information systems in small and medium-sized enterprises: a framework and empirical analysis. Measuring Business Excellence. 10(4): 8-21.

Sharma, M.K. and Bhagwat, R. 2007. Performance measurement system: case studies from SMEs in India. International Journal of Productivity and Quality Management. 2(4): 475509.

Sharma, M.K., Bhagwat, R. and Dangayach, G.S. 2005. Practice of performance measurement: experience from Indian SMEs. International Journal of Globalization and Small Business. 1(2): 183-213.

Smith, V. M. and Keoleian, G. A. 2004. The value of remanufactured engines. Journal of Industrial Ecology. 8(1-2): 193-221.

Tharumarajah, A., Wells, A.J. and Nemes, L. 1996. Comparison of the bionic, fractal and holonic manufacturing system concepts. International Journal of Computer Integrated Manufacturing. 9: 217-226.

Thierry, M. et al. 1995. Strategic issues in product recovery management. California Management Review. 37(2): 114-137.

Thomas Craig. 2003. 4PL versus 3PL-a business process outsourcing option for international supply chain management. World Wide Shipping. 4: 47-60.

Thomas, D. J. and Griffin, P. M. 1996. Coordinated supply chain management. European Journal of Operational Research. 94: 1-15.

Tirpak, T.M. and Daniel, S.M., LaLonde, J.D. and Davis, W.J. 1992. A note on a fractal architecture for modeling and controlling flexible manufacturing systems. IEEE Transactions on Systems, Man, and Cybernetics. 22: 564-567.

V.D.R. Guide Jr, V. Jayaraman, and J.D. Linton. 2003. Building contingency planning for closedloop supply chains with product recovery. Journal of Operations Management. 21(3): 
259-279.

Wang, D-K http://www.implight.net/node/4659

Wang, F-B 2005. Holography applied in supply chain management. System Engineering(in Chinese). 23: 8-12.

Wang, S. 2007. Keep improving university physics. Jinan: Shangdong University Publishing House.

Warnecke, H.J. 1993. The Fractal Company: a Revolution in Corporate Culture. Springer-Verlag. Berlin.

Williamson, O. E. 1991. Comparative economic organization: the analysis of discrete structural alternatives. Administrative Science Quarterly. 36: 269-296.

Wu, T. 2001. Research on Self-organization Methodology. Tsinghua University Publising Company. Beijing.

Yan, J. et al. 2003. Application of entropy in measuring the complexity of supply chain. Mechanical Science and Technology. 22(5):692-695.

Zhang, Y.G. and Li, W.S. 2004. Application of dissipative structure theory in supply chain management. Logistics Technology. 7: 42-45. 
